\title{
R-parity violation in SU(5)
}

\section{Borut Bajc $^{a}$ and Luca Di Luzio ${ }^{b}$}

\author{
${ }^{a}$ Department of Theoretical Physics, J. Stefan Institute, \\ Jamova cesta 39, 1000 Ljubljana, Slovenia \\ ${ }^{b}$ Dipartimento di Fisica, Università di Genova and INFN, Sezione di Genova, \\ via Dodecaneso 33, 16159 Genova, Italy \\ E-mail: borut.bajc@ijs.si, luca.di.luzio@ge.infn.it
}

ABSTRACT: We show that judiciously chosen R-parity violating terms in the minimal renormalizable supersymmetric SU(5) are able to correct all the phenomenologically wrong mass relations between down quarks and charged leptons. The model can accommodate neutrino masses as well. One of the most striking consequences is a large mixing between the electron and the Higgsino. We show that this can still be in accord with data in some regions of the parameter space and possibly falsified in future experiments.

KeYwords: Supersymmetric Standard Model, GUT

ARXIV EPRINT: 1502.07968 


\section{Contents}

1 Introduction and outline 1

2 The RPV SU(5) 4

2.1 The issue of the doublet basis 5

$\begin{array}{ll}2.2 & \text { The color triplet mass eigenstates }\end{array}$

2.3 The charged lepton mass eigenstates 8

2.4 How to avoid Yukawa unification 8

2.5 A numerical example 9

$\begin{array}{lll}2.6 & \text { The trilinear RPV couplings } & 10\end{array}$

3 Phenomenology 11

$\begin{array}{lll}3.1 & \text { Proton decay and unification constraints } & 12\end{array}$

$\begin{array}{ll}3.2 & \text { Electroweak symmetry breaking } \\ 3.34\end{array}$

$\begin{array}{lll}3.3 & \text { Neutrino masses } & 15\end{array}$

$\begin{array}{lll}\text { 3.3.1 Tree-level seesaw from RPV interactions } & 15\end{array}$

$\begin{array}{lll}\text { 3.3.2 } & \text { One-loop contributions from RPV couplings } & 15\end{array}$

$\begin{array}{ll}\text { 3.3.3 Seesaw from GUT-scale mediators } & 17\end{array}$

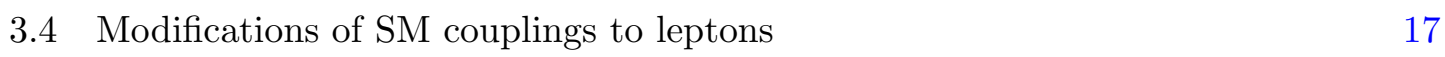

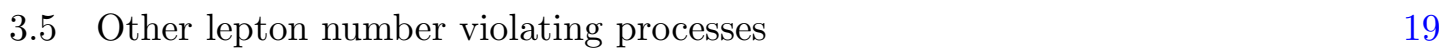

$\begin{array}{ll}3.6 & \text { Lepton flavour violation }\end{array}$

$\begin{array}{ll}3.7 & \text { Gravitino dark matter }\end{array}$

4 Discussion and conclusions $\quad 22$

$\begin{array}{lr}\text { A Perturbative diagonalization } & 25\end{array}$

\section{Introduction and outline}

$\mathrm{SU}(5)$ is the minimal and the simplest among supersymmetric grand unified theories (GUTs) [1]. It is thus of particular interest to test it in detail. In this work we will stick to its minimal renormalizable version with three matter copies of $\overline{5} \oplus 10$ and Higgs supermultiplets in $5 \oplus \overline{5} \oplus 24$. In fact, by allowing either non-renormalizable operators or extra superfields, many new unknown parameters enter in the superpotential thus making the model unpredictive.

Yet, it is well known that the minimal renormalizable SU(5) GUT suffers from two main drawbacks. First, it predicts the equality at the GUT scale of the down quark and charged lepton masses ( $i=1,2,3$ runs over generations)

$$
\frac{m_{D}^{i}}{m_{E}^{i}}=1
$$


where $\left(m_{D}^{1}, m_{D}^{2}, m_{D}^{3}\right)=\left(m_{d}, m_{s}, m_{b}\right)$ and $\left(m_{E}^{1}, m_{E}^{2}, m_{E}^{3}\right)=\left(m_{e}, m_{\mu}, m_{\tau}\right)$. While, running those masses from their $m_{Z}$ values up to the GUT scale and assuming (as an example) lowscale minimal supersymmetric standard model (MSSM) and low $\tan \beta$, gives (see e.g. [2])

$$
\left(\frac{m_{d}}{m_{e}}, \frac{m_{s}}{m_{\mu}}, \frac{m_{b}}{m_{\tau}}\right) \approx(2.6,0.23,0.81)
$$

The discrepancies are of order one, and so cannot be easily accounted for without changing the theory, for example its physical content. The second problem is the absence of neutrino masses, similarly as in the standard model (SM).

The issue of charged fermion masses in minimal renormalizable $\mathrm{SU}(5)$ can be solved by large supersymmetry (susy) breaking threshold corrections [3-12]. The prize to pay, however, is large $A$-terms which make the MSSM vacuum metastable [13]. Also, this does not bring any new ingredient for the solution of the neutrino mass problem.

In this work, we want to take instead an orthogonal approach. We neglect the contribution of susy threshold corrections and investigate whether the fermion mass ratio problem can be fixed by R-parity violating (RPV) [14] couplings in the SU(5) model. This idea has been first proposed long ago [15] (for some other works in this direction see for example $[16,17])$, but never systematically worked out. We will show that R-parity violation can correct all the wrong mass relations (1.1). This will immediately open up a solution also for the neutrino mass problem. Let us now briefly describe the idea, while the details will be worked out in the body of the paper.

It has been long known that giving up the minimal field content and allowing for extra vector-like matter fields it is possible to correct the $\mathrm{SU}(5)$ fermion mass relations (for an incomplete list of references see [2, 18-29]). As we will show later, the mixing of $d_{i}^{c}\left(L_{i}\right)$ with an extra vector-like color triplet (weak doublet) by angle $\theta_{D}^{i}\left(\theta_{E}^{i}\right)$ changes relation (1.1) into

$$
\frac{m_{D}^{i}}{m_{E}^{i}}=\frac{\cos \theta_{D}^{i}}{\cos \theta_{E}^{i}}
$$

However, we do not want to enlarge the field content of the model. An obvious (and well known) candidate for a vector-like pair is provided in the MSSM by the two Higgs doublets with bilinear RPV terms [30-34]. According to (1.3) with $\theta_{D}^{i}=0$ the mass ratio can only increase, so bilinear R-parity violation can be useful in the MSSM just for the first generation (see eq. (1.2)).

The next logical possibility is to allow also for color triplets $d_{i}^{c}$ to mix with the heavy SU(5) partners of the MSSM Higgses. At first glance this idea looks hopeless, since the mixing would induce the trilinear RPV couplings $\lambda^{\prime}$ and $\lambda^{\prime \prime}$ (cf. the superpotential in eq. (2.49)) from the $\mathrm{SU}(5)$ Yukawas after rotation. This would make the proton to decay too fast, since the $d=4$ proton decay amplitude is proportional to $\lambda^{\prime} \lambda^{\prime \prime}$ and suppressed just by the susy breaking scale. Moreover, SU(5) symmetry at the renormalizable level predicts for the RPV trilinear couplings (before rotation)

$$
\lambda=\lambda^{\prime}=\lambda^{\prime \prime}
$$


so that it seems impossible to disentangle $\lambda^{\prime}$ from $\lambda^{\prime \prime}$. However (1.4) is valid in the original (flavour) basis, and not in the mass eigenbasis. Since the rotation of quarks $d_{i}^{c}$ with the heavy color anti-triplet $\bar{T}$ makes the mass and flavour eigenbasis different, we can avoid (1.4). At this point, special care must be taken to cancel $\lambda^{\prime \prime}=0$, effectively preserving the baryon number below the GUT scale. This can be obtained by taking a very specific value of the trilinear RPV couplings. As we will see, the requirement of $\lambda^{\prime \prime}=0$ will uniquely determine the other trilinear RPV couplings as a function of the mixings.

From eq. (1.3) it is clear that only the relative misalignment between doublet and triplet rotations matters for the correction of the mass eigenvalues. Hence, we will take the additional simplifying assumption that at a given generation $i$ either the quark $\theta_{D}^{i}$ or the lepton $\theta_{E}^{i}$ angle contributes, but not both. This will then uniquely determine the mixings (i.e. the angles $\theta_{D}^{i}$ and $\theta_{E}^{i}$ ). By comparing (1.2) with (1.3) we conclude that $d^{c}$ quarks of the second and third generation will mix with the heavy triplet, while only the first generation lepton will require a mixing with the Higgs doublet. We will hence have

$$
\frac{m_{d}}{m_{e}}=\frac{1}{\cos \theta_{E}^{1}}, \quad \frac{m_{s}}{m_{\mu}}=\cos \theta_{D}^{2}, \quad \frac{m_{b}}{m_{\tau}}=\cos \theta_{D}^{3} .
$$

In the conclusions we will shortly comment on what happens if we relax these assumptions.

The resulting model turns out to be very much constrained. Not only one needs to do more than the usual single doublet-triplet fine-tuning, the original choice of the trilinear couplings must also magically combine in order to project to vanishing baryon number violating couplings after triplet rotation. Also, large lepton number violating couplings will induce tree and loop order neutrino masses, which will typically be too large unless under special conditions. We will not even attempt to understand or explain all these fortuitous relations among model parameters. But we will (shamelessly) use such possibility whenever needed by experimental data. In order to accommodate all these constraints our soft terms will not be subject to $\mathrm{SU}(5)$ invariant boundary condition at the GUT scale. We will hence assume that susy breaking is mediated below the GUT scale (for more comments on that see section 4 ). This exercise must be thus interpreted as a purely phenomenological possibility in order to avoid various constraints already in the minimal SU(5) model, and not as a proposal for a theoretically attractive theory.

In spite of this, or better, because of this, the model predicts a phenomenologically very interesting situation of a large mixing between the electron (neutrino) and the charged (neutral) Higgsino. The seemingly ad-hoc assumption of only quark or lepton mixing in the same generation will at this point help in avoiding strong phenomenological constraints due to large (order one) lepton number violating couplings present in the low-energy MSSM Lagrangian. In particular, we will see that the tiny neutrino masses predict in this scenario a fixed (negative) ratio between the wino and bino masses, provided they are not much larger than the sfermion masses. Finally, the same large RPV couplings only allow a slowly decaying gravitino lighter than about $10 \mathrm{MeV}$ as a dark matter (DM) candidate.

The paper is organised as follows: in section 2 we discuss the general structure of the RPV SU(5) model and show how RPV interactions can correct the wrong mass relations of the original SU(5) model. Most of section 3 is instead devoted to checking whether the 
required amount of R-parity violation is still allowed by data. In particular, we discuss proton decay bounds, electroweak symmetry breaking, neutrino masses, modifications of SM couplings to leptons, lepton number and lepton flavour violating processes and gravitino DM. We conclude in section 4 by recalling the main predictions of the model, while more technical details on the diagonalization of the relevant mass matrices are collected in appendix A.

\section{The RPV SU(5)}

The field content of the minimal $\mathrm{SU}(5)$ model is given by $5, \overline{5}_{\alpha}(\alpha=0,1,2,3), 10_{i}(i=$ $1,2,3)$ and 24 . The decomposition of the $\mathrm{SU}(5)$ supermultiplets under the SM gauge quantum numbers reads

$$
5=\left(\begin{array}{c}
T \\
H_{u}
\end{array}\right), \quad \overline{5}_{\alpha}=\left(\begin{array}{c}
\overline{3} \\
\overline{2}
\end{array}\right)_{\alpha}, \quad 10_{i}=\left(\begin{array}{cc}
\epsilon_{3} u^{c} & Q \\
-Q^{T} & -\epsilon_{2} e^{c}
\end{array}\right)_{i},
$$

where $\epsilon_{3}\left(\epsilon_{2}\right)$ schematically denotes the Levi-Civita tensor in the $\mathrm{SU}(3)(\mathrm{SU}(2))$ space and for the adjoint (which also spontaneously breaks SU(5) into the SM gauge group)

$$
24=\left(V+\frac{\phi_{(1,1)_{0}}}{\sqrt{30}}\right)\left(\begin{array}{cc}
2 & 0 \\
0 & -3
\end{array}\right)+\left(\begin{array}{cc}
\phi_{(8,1)_{0}} & \phi_{(\overline{3}, 2)_{5 / 6}} \\
\phi_{(3, \overline{2})_{-5 / 6}} & \phi_{(1,3)_{0}}
\end{array}\right) .
$$

The indices of $\phi$ stand for the SM gauge quantum numbers, while the part proportional to $V$ denotes the GUT vacuum expectation value (vev). The most general renormalizable superpotential is

$$
\begin{aligned}
W_{\mathrm{SU}(5)}= & \overline{5}_{\alpha}\left(M_{\alpha}+\eta_{\alpha} 24\right) 5+\frac{1}{2} \Lambda_{\alpha \beta k} \overline{5}_{\alpha} \overline{5}_{\beta} 10_{k} \\
& +Y_{i j}^{10} 10_{i} 10_{j} 5+\frac{M_{24}}{2} \operatorname{Tr} 24^{2}+\frac{\lambda_{24}}{3} \operatorname{Tr} 24^{3},
\end{aligned}
$$

where SU(5) contractions are understood. In particular, $\Lambda_{\alpha \beta k}=-\Lambda_{\beta \alpha k}$ and $Y_{i j}^{10}=Y_{j i}^{10}$. The usual R-parity conserving (RPC) case

$$
\begin{aligned}
W_{\mathrm{SU}(5)}^{\mathrm{RPC}}= & \overline{5}_{0}\left(M_{0}+\eta_{0} 24\right) 5+Y_{j k}^{5} \overline{5}_{0} \overline{5}_{j} 10_{k} \\
& +Y_{i j}^{10} 10_{i} 10_{j} 5+\frac{M_{24}}{2} \operatorname{Tr} 24^{2}+\frac{\lambda_{24}}{3} \operatorname{Tr} 24^{3},
\end{aligned}
$$

is recovered in the limit

$$
M_{\alpha}=M_{0} \delta_{\alpha 0}, \quad \eta_{\alpha}=\eta_{0} \delta_{\alpha 0}, \quad \Lambda_{\alpha \beta k}=Y_{j k}^{5}\left(\delta_{\alpha 0} \delta_{\beta j}-\delta_{\alpha j} \delta_{\beta 0}\right)
$$

The terms in the second line of eq. (2.4) and eq. (2.3) coincide: $Y^{10}$ is responsible for the up-quark masses, while $M_{24}$ and $\lambda_{24}$ participate to the GUT symmetry breaking and are related by the minimum equation to the $\mathrm{SU}(5)$ breaking vev in eq. (2.2) via the relation $V=M_{24} / \lambda_{24}$. Moreover, in the RPC case $Y^{5}$ leads to the usual Yukawa unification condition (1.1) which we want to correct with the more general superpotential in (2.3). 
Let us now focus on the first line of eq. (2.3). From the first term we see that one combination of $\overline{5}_{\alpha}$ gets a vector-like mass with 5 . Physically we know that such a mass has to be large in the triplet sector and light in the doublet one. This can be achieved thanks to $\mathrm{SU}(5)$ breaking via the vev contribution in eq. (2.2). Then the mass terms in the doublet-triplet sector of the superpotential become

$$
W_{\text {mass }}=\overline{3}_{\alpha} \mathcal{M}_{\alpha} T+\overline{2}_{\alpha} \mu_{\alpha} H_{u}
$$

where

$$
\begin{aligned}
\mathcal{M}_{\alpha} & =M_{\alpha}+2 \eta_{\alpha} V, \\
\mu_{\alpha} & =M_{\alpha}-3 \eta_{\alpha} V .
\end{aligned}
$$

The doublet-triplet splitting (assuming low-energy susy) means the following:

$$
\mu_{\alpha} \lesssim \mathcal{O}\left(m_{W}\right)
$$

for all $\alpha=0,1,2,3$, while

$$
\mathcal{M}_{\alpha}=\mathcal{O}\left(M_{\mathrm{GUT}}\right)
$$

for at least one $\alpha$. Notice that while in the usual RPC case one fine-tuning is enough, in the generic RPV case four fine-tunings are needed in order to satisfy eq. (2.9) for all four possible choices of $\alpha$.

Finally, the terms in $\Lambda_{\alpha \beta k}$ contain, on top of the above mentioned Yukawas, the trilinear RPV couplings which will be discussed in section 2.6.

\subsection{The issue of the doublet basis}

Since in this setup there is no real difference between the four doublet superfields $\overline{2}_{\alpha}=$ $\left(N_{\alpha}, E_{\alpha}\right)^{T}$, what do we mean by the names (s)neutrino, charged or neutral Higgs(ino) and charged (s)lepton? In other words, what is the difference between neutral Higgs-sneutrino, neutral Higgsino-neutrino, charged Higgs-slepton and charged Higgsino-charged lepton? Although the results can always be written in a basis-independent way [35, 36] and so these names are strictly speaking not really necessary, we will still define such names for the sake of clarity.

We will choose a convenient basis, in which only one among the SM doublets $\overline{2}_{\alpha} \subset \overline{5}_{\alpha}$ (let it be the one with index $\alpha=0$ ) gets a nonzero vev $v_{d}$. This can be obtained by an $\mathrm{SU}(4)$ rotation of the $\overline{5}_{\alpha}$ which affects the relations (2.7)-(2.8) as well. One could argue that the new, rotated, $M_{\alpha}$ and $\eta_{\alpha}$ cannot be completely arbitrary, since the vevs themselves depend on them. However, it is not hard to imagine (and we will show it in more detail in section 3.2) that the freedom in the choice of soft terms allows us to consider $M_{\alpha}$ and $\eta_{\alpha}$ arbitrary with $\left\langle\overline{5}_{i}\right\rangle=0$. Since we will not employ any particular spectrum of the soft terms, this is what we can (and will) do.

In particular, there are essentially four classes of fields we have to specify: the neutral bosons, the neutral fermions, the charged bosons and the charged fermions. These are fixed in the following way: 
- The flavour basis of neutral bosons is defined such that the sneutrinos' vevs vanish:

$$
\left\langle\tilde{\nu}_{i}\right\rangle=0, \quad i=1,2,3
$$

i.e. we define the neutral Higgs vevs as in the RPC case:

$$
\left\langle H_{u}^{0}\right\rangle \equiv v_{u}=v \sin \beta, \quad\left\langle H_{d}^{0}\right\rangle \equiv v_{d}=v \cos \beta,
$$

where $v=246 \mathrm{GeV}$. More details about the electroweak symmetry breaking sector and the composition of the lightest Higgs boson in terms of the flavour basis can be found in section 3.2 .

- The neutral fermion mass matrix is incorporated into the neutralino quadratic part of the lagrangian (see e.g. [37]):

$$
\begin{aligned}
& \mathcal{L}_{N}=-\frac{1}{2}\left(\begin{array}{lllll}
\tilde{B}^{0} & \tilde{W}^{0} & \tilde{H}_{u}^{0} & \tilde{H}_{d}^{0} & \nu_{i}
\end{array}\right) \\
& \left(\begin{array}{ccccc}
M_{1} & 0 & g^{\prime} v_{u} / 2 & -g^{\prime} v_{d} / 2 & 0 \\
0 & M_{2} & -g v_{u} / 2 & g v_{d} / 2 & 0 \\
g^{\prime} v_{u} / 2 & -g v_{u} / 2 & 0 & -\mu_{0} & -\mu_{k} \\
-g^{\prime} v_{d} / 2 & g v_{d} / 2 & -\mu_{0} & \eta_{0} \eta_{0} v_{u}^{2} / M_{\text {seesaw }} & \eta_{0} \eta_{k} v_{u}^{2} / M_{\text {seesaw }} \\
0 & 0 & -\mu_{i} & \eta_{i} \eta_{0} v_{u}^{2} / M_{\text {seesaw }} & \eta_{i} \eta_{k} v_{u}^{2} / M_{\text {seesaw }}
\end{array}\right)\left(\begin{array}{c}
\tilde{B}^{0} \\
\tilde{W}^{0} \\
\tilde{H}_{u}^{0} \\
\tilde{H}_{d}^{0} \\
\nu_{k}
\end{array}\right)
\end{aligned}
$$

where we added the $4 \times 4$ lower-right block as the seesaw contribution from the SM singlet $(1,1)_{0}[38-42]$ and weak triplet $(1,3)_{0}[43]$ states living in 24 , and

$$
\frac{1}{M_{\text {seesaw }}}=\frac{3}{10} \frac{1}{M_{(1,1)_{0}}}+\frac{1}{2} \frac{1}{M_{(1,3)_{0}}}=-\frac{2}{5} \frac{1}{M_{24}},
$$

with $M_{24}$ denoting the superpotential parameter defined in eq. (2.3), $M_{(1,3)_{0}}=-5 M_{24}$ and $M_{(1,1)_{0}}=-M_{24}$.

It is clear from (2.13) that in the flavour basis $\tilde{H}_{d}^{0}$ is the fermionic superpartner of $H_{d}^{0}$ that gets the vev in (2.12). The mass basis is obtained by diagonalizing the matrix in eq. (2.13) and neutrinos are the three lightest eigenstates.

- The charged fermions are part of the chargino sector (see e.g. [37]):

$$
\mathcal{L}_{C}=-\left(\begin{array}{lll}
\tilde{W}^{-} & \tilde{H}_{d}^{-} & e_{i}
\end{array}\right)\left(\begin{array}{ccc}
M_{2} & g v_{u} / \sqrt{2} & 0 \\
g v_{d} / \sqrt{2} & \mu_{0} & 0 \\
0 & \mu_{i} & \Lambda_{0 i k} v_{d}
\end{array}\right)\left(\begin{array}{c}
\tilde{W}^{+} \\
\tilde{H}_{u}^{+} \\
e_{k}^{c}
\end{array}\right) .
$$

$\tilde{H}_{d}^{-}$and $e_{i}$ are the weak partners of the previously defined $\tilde{H}_{d}^{0}$ and $\nu_{i}$, respectively. In particular, the charged lepton mass eigenstates correspond to the three lightest eigenvalues of the matrix in eq. (2.15).

- Finally the charged bosons: in the flavour basis they are just the $\mathrm{SU}(2)$ partners of the neutral bosons defined through (2.11) and (2.12), or, equivalently, the bosonic superpartners of the charged fermions defined in (2.15). We will denote them by $H_{d}^{-}$and $\tilde{e}_{i}$. 
This quadratic part of the Lagrangian, plus the analogous one for color triplets in (2.17), is RPC if $\mathcal{M}_{i}=\mu_{i}=0$. Of course, the whole Lagrangian, or even this part of it at higher loops, is not RPC due to nonzero trilinear terms, but in the basis we use, $\left\langle\tilde{\nu}_{i}\right\rangle=0$, these trilinear terms do not appear in the mass matrices at the tree order.

At this point, we are still free to rotate in the $3 \times 3$ subspace and we use this freedom to diagonalize the sub-matrix matrix

$$
\Lambda_{0 i k}=\delta_{i k} d_{k}
$$

Consequently, eqs. (2.7)-(2.8) get rotated as well, but we will not keep track of it.

\subsection{The color triplet mass eigenstates}

The mass matrix for color triplets comes from the first term in (2.6) and the last term in the first line of $(2.3)$

$$
\mathcal{L}_{3}=-\left(\begin{array}{ll}
\overline{3}_{0} & \overline{3}_{i}
\end{array}\right)\left(\begin{array}{cc}
\mathcal{M}_{0} & 0 \\
\mathcal{M}_{i} & \Lambda_{0 i k} v_{d}
\end{array}\right)\left(\begin{array}{c}
T \\
Q_{k}
\end{array}\right) .
$$

The states $\overline{3}_{\alpha}$ are still in the flavour basis. Let us rotate them into the mass eigenstates $\left(\bar{T}, d_{k}^{c}\right)$. Since $\mathcal{M}_{\alpha}=\mathcal{O}\left(M_{\mathrm{GUT}}\right) \gg \Lambda_{0 i k} v_{d}=\mathcal{O}\left(m_{W}\right)$, we can easily disentangle the single heavy state $\bar{T}$ from the light ones $d_{k}^{c}$ :

$$
\overline{3}_{\alpha}=\left(\bar{T} d^{c}\right)_{\beta} U_{\beta \alpha},
$$

where the matrix $U$ projects the triplet states into the heavy direction

$$
\begin{aligned}
\mathrm{U}\left(1, x_{i}\right)^{T} & \propto(1,0,0,0)^{T}, \\
x_{i} & =\mathcal{M}_{i} / \mathcal{M}_{0} .
\end{aligned}
$$

Assuming everything real for simplicity we have (see for example [26])

$$
U=\left(\begin{array}{cc}
U_{00} & U_{0 j} \\
U_{i 0} & U_{i j}
\end{array}\right),
$$

where

$$
\begin{aligned}
U_{00} & =\frac{1}{\sqrt{1+\vec{x}^{2}}}, \\
U_{0 j} & =\frac{x_{j}}{\sqrt{1+\vec{x}^{2}}}, \\
U_{i 0} & =-\frac{x_{i}}{\sqrt{1+\vec{x}^{2}}}, \\
U_{i j} & =\delta_{i j}-\frac{x_{i} x_{j}}{\sqrt{1+\vec{x}^{2}}\left(\sqrt{1+\vec{x}^{2}}+1\right)} .
\end{aligned}
$$

Then the light $3 \times 3$ mass matrix (of the down quarks) is

$$
\left(M_{D}\right)_{i j}=U_{i j}(x) d_{j} v_{d}
$$


Notice that $U_{i j}$ is not unitary, since it is just the $3 \times 3$ sub-matrix of the $4 \times 4$ unitary $U_{\alpha \beta}$. This implies that the mass eigenvalue

$$
m_{D}^{j}(x) \leq m_{D}^{j}(0)=d_{j} v_{d},
$$

for any $\vec{x}$.

\subsection{The charged lepton mass eigenstates}

In order to get the three lightest eigenvalues of the chargino mass matrix it turns out to be a good approximation to consider the gaugino decoupling limit. This will be numerically confirmed in section 2.5. In this case what remains in eq. (2.15) is

$$
\left(\begin{array}{cc}
\mu_{0} & 0 \\
\mu_{i} & \Lambda_{0 i k} v_{d}
\end{array}\right),
$$

which is analogous to (2.17). Although the Higgsino mass is presumably much lighter than the GUT scale, it is still much heavier than the light charged leptons, so a similar rotation as in the case of the triplets can be used to integrate out the heavy Higgsino. The light charged lepton mass matrix is thus in this limit

$$
\left(M_{E}\right)_{i j}=U_{i j}(y) d_{j} v_{d}
$$

with

$$
y_{i}=\mu_{i} / \mu_{0}
$$

As before, we have

$$
m_{E}^{j}(x) \leq m_{E}^{j}(0)=d_{j} v_{d},
$$

for any $\vec{y}$.

\subsection{How to avoid Yukawa unification}

We are interested in the correlation between down quarks (eq. (2.26)) and charged leptons (eq. (2.29)). It is known, see for example [2] and references therein, that with arbitrary $x_{i}, y_{i}$ and $d_{i}$, one can fit all down quark and charged lepton masses. In fact, defining the Yukawa $\lambda=m / v_{d}$, one finds in the hierarchical limit $d_{1} \ll d_{2} \ll d_{3}$

$$
\begin{aligned}
\lambda_{d} & =\frac{1}{\sqrt{1+x_{1}^{2}}} d_{1}, & \lambda_{e} & =\frac{1}{\sqrt{1+y_{1}^{2}}} d_{1}, \\
\lambda_{s} & =\frac{\sqrt{1+x_{1}^{2}}}{\sqrt{1+x_{1}^{2}+x_{2}^{2}}} d_{2}, & \lambda_{\mu} & =\frac{\sqrt{1+y_{1}^{2}}}{\sqrt{1+y_{1}^{2}+y_{2}^{2}}} d_{2}, \\
\lambda_{b} & =\frac{\sqrt{1+x_{1}^{2}+x_{2}^{2}}}{\sqrt{1+x_{1}^{2}+x_{2}^{2}+x_{3}^{2}}} d_{3}, & \lambda_{\tau} & =\frac{\sqrt{1+y_{1}^{2}+y_{2}^{2}}}{\sqrt{1+y_{1}^{2}+y_{2}^{2}+y_{3}^{2}}} d_{3} .
\end{aligned}
$$

From these equations it is clear that the most economical way to get (1.2) is to take $x_{1}=0$ (no mixing of the heavy color triplet with the first generation down quark) and $y_{2}=y_{3}=0$ (no mixing of the Higgsino with the second and third generation lepton). 
Before ending, we want to make a connection with the notation of eq. (1.3). This can be done by defining the angles

$$
\begin{aligned}
\tan \theta_{D}^{1} & =x_{1}, & \tan \theta_{E}^{1} & =y_{1}, \\
\tan \theta_{D}^{2} & =\frac{x_{2}}{\sqrt{1+x_{1}^{2}}}, & \tan \theta_{E}^{2} & =\frac{y_{2}}{\sqrt{1+y_{1}^{2}}}, \\
\tan \theta_{D}^{3} & =\frac{x_{3}}{\sqrt{1+x_{1}^{2}+x_{2}^{2}}}, & \tan \theta_{E}^{3} & =\frac{y_{3}}{\sqrt{1+y_{1}^{2}+y_{2}^{2}}} .
\end{aligned}
$$

Then the masses are

$$
m_{D, E}^{i}=v_{d} d_{i} \cos \theta_{D, E}^{i},
$$

from which eq. (1.3) follows.

\subsection{A numerical example}

As a numerical benchmark let us consider the case of MSSM with $\tan \beta=7$ and low susy scale. From the experimental values at $m_{Z}$ one can use the renormalization group equations (RGEs) to get the charged lepton and down quark Yukawa couplings at the GUT scale [2]

$$
\begin{aligned}
& \left(\lambda_{e}^{\exp }, \lambda_{\mu}^{\exp }, \lambda_{\tau}^{\exp }\right)=(0.000013,0.0028,0.047), \\
& \left(\lambda_{d}^{\exp }, \lambda_{s}^{\exp }, \lambda_{b}^{\exp }\right)=(0.000034,0.00063,0.038) .
\end{aligned}
$$

As we saw in the previous paragraph, the Yukawas can only diminish if a mixing with an extra vector-like $L-\bar{L}$ or $d^{c}-\overline{d^{c}}$ is introduced. Since from (2.39)-(2.40) $\lambda_{e}^{\exp }<\lambda_{d}^{\exp }$, but $\lambda_{\mu}^{\exp }>\lambda_{s}^{\exp }$ and $\lambda_{\tau}^{\exp }>\lambda_{b}^{\exp }$, the minimal option is to keep $\lambda_{d}, \lambda_{\mu}$ and $\lambda_{\tau}$ unaltered, i.e.

$$
\begin{aligned}
& d_{1}=\lambda_{d}=\lambda_{d}^{\exp }=0.000034, \\
& d_{2}=\lambda_{\mu}=\lambda_{\mu}^{\exp }=0.0028, \\
& d_{3}=\lambda_{\tau}=\lambda_{\tau}^{\exp }=0.047,
\end{aligned}
$$

but correct (diminish) $\lambda_{e}=d_{1}, \lambda_{s}=d_{2}, \lambda_{b}=d_{3}$ to $\lambda_{e}^{\exp }, \lambda_{s}^{\exp }, \lambda_{b}^{\exp }$, respectively, by properly choosing the various $x_{i}, y_{i}$ (see eqs. (2.32)-(2.34)):

$$
\begin{aligned}
x_{1} & =0, \\
x_{2} & =\sqrt{\left(\lambda_{\mu}^{\exp } / \lambda_{s}^{\exp }\right)^{2}-1}=4.3, \\
x_{3} & =\left(\lambda_{\mu}^{\exp } / \lambda_{s}^{\exp }\right) \sqrt{\left(\lambda_{\tau}^{\exp } / \lambda_{b}^{\exp }\right)^{2}-1}=3.2, \\
y_{1} & =\sqrt{\left(\lambda_{d}^{\exp } / \lambda_{e}^{\exp }\right)^{2}-1}=2.4, \\
y_{2,3} & =0
\end{aligned}
$$

Notice that we fit all the masses at $M_{\mathrm{GUT}}$. Although this is a correct procedure for the quarks, since we are integrating out the heavy (GUT scale) color triplet, the lepton (electron) corrections should be determined in principle at low energy, when the Higgsino is integrated out. But since the RGEs for the light Yukawas are essentially linear $\left(d \lambda_{e} / d t \propto\right.$ $\lambda_{e}$ ), the result is practically the same. 
As a final remark, the r.h.s. of eq. (2.32) for the electron mass is only approximate, since the full mass matrix in eq. (2.15) contains mixings with gauginos as well. It is easy to check its consistency. The result is that the error by taking the approximate formula (2.32) is always below $2 \%$ for $M_{2}>1 \mathrm{TeV}$.

\subsection{The trilinear RPV couplings}

Let us define the RPV superpotential of the low-energy MSSM effective theory as

$$
W_{\mathrm{RPV}}=H_{u} \mu_{i} L_{i}+\frac{1}{2} \lambda_{i j k}^{\prime \prime} d_{i}^{c} d_{j}^{c} u_{k}^{c}+\frac{1}{2} \lambda_{i j k} L_{i} L_{j} e_{k}^{c}+\lambda_{i j k}^{\prime} d_{i}^{c} L_{j} Q_{k} .
$$

The trilinear RPV couplings are then obtained by decomposing the SU(5) superpotential (2.3) under the SM group and by matching it with eq. (2.49). This operation yields

$$
\begin{aligned}
\lambda_{i j k}^{\prime \prime} & =U_{i \alpha} U_{j \beta} \Lambda_{\alpha \beta k}, \\
\lambda_{i j k}^{\prime} & =U_{i \alpha} \Lambda_{\alpha j k}, \\
\lambda_{i j k} & =\Lambda_{i j k} .
\end{aligned}
$$

By enforcing the safe condition ${ }^{1}$

$$
0=\lambda_{i j k}^{\prime \prime}=\left(U_{i 0} U_{j n}-U_{\mathrm{in}} U_{j 0}\right) \Lambda_{0 n k}+U_{i l} U_{j n} \Lambda_{l n k},
$$

we can calculate the other trilinear couplings. To this end, we use the choice of basis in (2.16), the explicit form of $U$ in (2.21) and the relation

$$
\left(\delta_{i k}+\frac{x_{i} x_{k}}{1+\sqrt{1+\vec{x}^{2}}}\right)\left(\delta_{k j}-\frac{x_{k} x_{j}}{\sqrt{1+\vec{x}^{2}}\left(1+\sqrt{1+\vec{x}^{2}}\right)}\right)=\delta_{i j},
$$

which allows to compute the inverse of $U$. Hence, after some algebra we obtain

$$
\lambda_{i j k}=\left(x_{i} \delta_{j k}-x_{j} \delta_{i k}\right) d_{k},
$$

or explicitly (for the numerical example discussed in section 2.5)

$$
\begin{aligned}
\lambda_{i j 3}=d_{3}\left(\begin{array}{ccc}
0 & 0 & x_{1} \\
0 & 0 & x_{2} \\
-x_{1} & -x_{2} & 0
\end{array}\right)_{i j} \rightarrow\left(\begin{array}{ccc}
0 & 0 & 0 \\
0 & 0 & 0.20 \\
0 & -0.20 & 0
\end{array}\right)_{i j}, \\
\lambda_{i j 2}=d_{2}\left(\begin{array}{ccc}
0 & x_{1} & 0 \\
-x_{1} & 0 & -x_{3} \\
0 & x_{3} & 0
\end{array}\right)_{i j} \rightarrow\left(\begin{array}{ccc}
0 & 0 & 0 \\
0 & 0 & -0.0088 \\
0 & 0.0088 & 0
\end{array}\right)_{i j}, \\
\lambda_{i j 1}=d_{1}\left(\begin{array}{ccc}
0 & -x_{2} & -x_{3} \\
x_{2} & 0 & 0 \\
x_{3} & 0 & 0
\end{array}\right)_{i j} \rightarrow\left(\begin{array}{ccc}
0 & -0.00014 & -0.00011 \\
0.00014 & 0 & 0 \\
0.00011 & 0 & 0
\end{array}\right)_{i j},
\end{aligned}
$$

\footnotetext{
${ }^{1}$ The exact condition $\lambda^{\prime \prime}=0$ is, strictly speaking, unnecessary. However, the most conservative bounds from matter stability require $\left|\lambda^{\prime} \lambda^{\prime \prime}\right|<10^{-10}$ for any flavour index and for superpartners around the TeV scale [44], while analogous bounds hold as well for the combinations $\left|\lambda \lambda^{\prime \prime}\right|$ and $\left|\mu_{i} / \mu_{0} \lambda^{\prime \prime}\right|$. Hence, in practice, large mixings in the triplet $\left(x_{i}\right)$ and doublet $\left(y_{i}\right)$ sectors require $\lambda^{\prime \prime} \approx 0$. For a recent discussion of baryonic R-parity violation in GUTs see e.g. [45].
} 
where we used for our fit $x_{1}=0$. The only relevant matrix element (i.e. $\propto d_{3}=\lambda_{\tau}$ ) is then $\lambda_{233}=-\lambda_{323}$.

Similarly, for the other trilinear term we get

$$
\lambda_{i j k}^{\prime}=\left(-x_{j} \delta_{i k}+\frac{x_{i} x_{j} x_{k}}{\sqrt{1+\vec{x}^{2}}\left(1+\sqrt{1+\vec{x}^{2}}\right)}\right) d_{k} .
$$

Even in this case the piece proportional to $\lambda_{\tau}$ never goes through the first generation, i.e. $\lambda_{i j k}^{\prime} \propto \lambda_{e}$ if any among $i, j, k$ equals 1 , since $x_{1}=0$. This is important, since in this way many dangerous processes, like for example neutrinoless double $\beta$ decay, get automatically suppressed (cf. sections 3.5-3.6). Numerically we get

$$
\begin{aligned}
& \lambda_{i 3 k}^{\prime} \rightarrow\left(\begin{array}{ccc}
-0.00011 & 0 & 0 \\
0 & -0.0042 & 0.059 \\
0 & 0.0035 & -0.11
\end{array}\right)_{i k} \\
& \lambda_{i 2 k}^{\prime} \rightarrow\left(\begin{array}{ccc}
-0.00014 & 0 & 0 \\
0 & -0.0056 & 0.079 \\
0 & 0.0046 & -0.14
\end{array}\right)_{i k} \\
& \lambda_{i 1 k}^{\prime} \rightarrow\left(\begin{array}{ccc}
0 & 0 & 0 \\
0 & 0 & 0 \\
0 & 0 & 0
\end{array}\right)_{i k} \text {. }
\end{aligned}
$$

To summarize, the $L_{1}$ lepton number is strongly broken by the $\mathcal{O}(1)$ parameter $\mu_{1} / \mu_{0}$, $L_{2}$ by the $\mathcal{O}(0.1)$ couplings $\lambda_{233}$ and $\lambda_{i 23}^{\prime}, i=2,3$, and $L_{3}$ by the $\mathcal{O}(0.1)$ values of $\lambda_{i 33}^{\prime}$, $i=2,3$. Neutrino masses are thus generically expected to be large (see section 3.3). On the other hand, baryon number is effectively preserved below the GUT scale, thanks to the condition $\lambda^{\prime \prime}=0$.

\section{Phenomenology}

To study the phenomenology, we have to define our low-energy effective theory which is the MSSM with specific RPV couplings. As we saw, the low-energy RPV parameters considered so far are strongly correlated. In general they are parametrized by $x_{i}\left(=\mathcal{M}_{i} / \mathcal{M}_{0}\right)$ and $y_{i}\left(=\mu_{i} / \mu_{0}\right)$. In order to simplify our analysis and minimize the corrections to be done, we assumed that the RPV parameters which make the fermion mass problem more severe are not present (i.e. $x_{1}=y_{2,3}=0$ ). Due to that we will limit our phenomenological analysis to the case

$$
\vec{\mu}=\left(\mu_{1}, 0,0\right) .
$$

Recall that the numerical values of the RPV couplings $\mu_{i} / \mu_{0}, \lambda_{i j k}$ and $\lambda_{i j k}^{\prime}$ are at this point all known (cf. eqs. (2.44)-(2.48), eqs. (2.56)-(2.58) and eqs. (2.60)-(2.62)), being determined by the requirement of vanishing baryon number violating couplings in the MSSM and the correct fit to fermion masses.

To study the phenomenological consequences of the model we also need to specify the other RPV couplings which did not enter in the analysis so far, but which can still have a 
strong phenomenological impact: the soft mass terms $B_{i}, m_{0 j}^{2}$ as well as the trilinears $A_{i j k}$, $A_{i j k}^{\prime}$ and $A_{i j k}^{\prime \prime}$. Since it is not our intent to do here a full phenomenological study of the most general case, but just to show the existence of a realistic model, we will take further simplifying assumptions: let

- the RPV bilinear soft terms point in the direction 1 , similarly as the $\mu_{i}$ in the superpotential

$$
\begin{aligned}
B_{i} & \propto \delta_{i 1}, \\
m_{0 i}^{2} & \propto \delta_{i 1} .
\end{aligned}
$$

Although one would be tempted to make both r.h.s. in (3.2) and (3.3) to vanish, electroweak symmetry breaking constraints do not allow such choice, see section 3.2 ;

- the RPV trilinear terms vanish

$$
A_{i j k}=A_{i j k}^{\prime}=A_{i j k}^{\prime \prime}=0
$$

We are now ready to study the phenomenology. We will first consider proton decay. Here there are two new issues compared to the RPC case. First, as we will see in the next section, an additional constraint must be taken into account in the unification analysis. Second, due to the huge sensitivity of proton decay to the exact value of $\lambda^{\prime \prime} \approx 0$, new decay channels might contribute as well. After that we will systematically go through leptonic RPV consequences.

\subsection{Proton decay and unification constraints}

Although we will not dwell too much on the proton decay issue, some remarks are due. Unification of gauge couplings [46-49] in the minimal renormalizable SU(5) model seems at odds with the experimental limits on proton decay if one assumes order TeV susy spectrum [50], albeit playing with the flavour structure of soft terms allows to solve the problem $[51,52]$. Another logical possibility is simply to increase the susy scale. Nowadays, following ugly experimental facts and neglecting beautiful theoretical ideas, this is not a taboo anymore. In the usual RPC case it is enough to increase the susy scale to the multi-TeV region for low $\tan \beta$ in order to get the $d=5$ proton decay channel under control $[53,54]$. The point is [54] that by increasing the susy scale the color-triplet mass rises as well due to gauge coupling unification constraints. On the other side, this reduces the combination of the heavy gauge boson mass squared times the mass parameter of the adjoint. The gauge boson mass cannot be too low due to the $d=6$ proton decay channel, but in the RPC case the mass of the adjoint can practically take any value and so can be diminished at will.

Once however R-parity conservation is abandoned, and the $\eta_{i}$ are of order one due to the doublet-triplet fine-tuning (2.7)-(2.8), the adjoint mass cannot be too small because it mediates the type I + III seesaw mechanism for neutrino masses (see eq. (2.13) and section 3.3.3), so it is bounded from below by around $10^{13} \mathrm{GeV}$. This means that we 
cannot increase the susy scale at will and so we may have some problem with proton decay constraints.

Let us now estimate these scales. Denoting by $m_{\tilde{f}}$ the common sfermion mass (taken also as the matching scale between SM and MSSM), by $m_{\lambda}$ the common gaugino mass, by $\mu$ the Higgsino mass $\left(\mu_{0} \approx \mu_{1}\right.$ ), by $M_{T}$ the heavy color triplet mass, by $M_{V}$ the heavy gauge boson mass (taken also as the matching scale between MSSM and SU(5)) and $M_{24}$ the common mass of the heavy adjoint fields (differences due to order one Clebsches are neglected), we can write the approximate relations [54, 55]

$$
\begin{aligned}
\left(\frac{M_{T}}{10^{15} \mathrm{GeV}}\right)^{6} & \approx\left(\frac{\mu}{1 \mathrm{TeV}}\right)^{5}, \\
\left(\frac{M_{V}}{10^{16} \mathrm{GeV}}\right)^{6}\left(\frac{M_{24}}{10^{16} \mathrm{GeV}}\right)^{3} & \approx\left(\frac{1 \mathrm{TeV}}{m_{\lambda}}\right)^{2},
\end{aligned}
$$

together with the experimental constraints from $d=5$ proton decay, $d=6$ proton decay, tree-level contribution to neutrino masses from the exchange of heavy mediators from the adjoint, and perturbativity, respectively:

$$
\begin{aligned}
\left(\frac{M_{T}}{10^{15} \mathrm{GeV}}\right)\left(\frac{m_{\tilde{f}}}{1 \mathrm{TeV}}\right)^{2}\left(\frac{1 \mathrm{TeV}}{\max \left(m_{\lambda}, \mu\right)}\right) \frac{1}{\tan \beta} & \gtrsim 10^{3}, \\
\left(\frac{M_{V}}{10^{16} \mathrm{GeV}}\right) & \gtrsim 1 / 3, \\
\left(\frac{M_{24}}{10^{16} \mathrm{GeV}}\right) & \gtrsim 10^{-3}, \\
\left(\frac{M_{T}}{10^{15} \mathrm{GeV}}\right)\left(\frac{10^{16} \mathrm{GeV}}{M_{V}}\right) & \lesssim 10 .
\end{aligned}
$$

Hence, we immediately find an upper (lower) limit on the gaugino (sfermion) masses:

$$
\begin{aligned}
& m_{\lambda} \lesssim 10^{6} \mathrm{TeV}, \\
& m_{\tilde{f}} \gtrsim 30 \mathrm{TeV} \sqrt{\tan \beta}\left(\Theta\left(\mu-m_{\lambda}\right)+\sqrt{m_{\lambda} / \mu} \Theta\left(m_{\lambda}-\mu\right)\right) .
\end{aligned}
$$

So, as an example, we can have at small $\tan \beta \approx 2$ a common but relatively high-susy scale

$$
m_{\lambda}, m_{\tilde{f}}, \mu \approx 60 \mathrm{TeV}
$$

with

$$
\begin{aligned}
M_{24} & \approx 10^{13.8} \mathrm{GeV}, \\
M_{V}, M_{T} & \approx 10^{16.5} \mathrm{GeV} .
\end{aligned}
$$

In such a case the $d=5$ proton decay channel is the leading one and to be seen soon.

In all other solutions, the susy spectrum must be split with possibly light Higgsino and/or gauginos. It has to be stressed though that all we said so far is valid at most as an order of magnitude estimate, so that factors of few are possible. 
Finally, let us notice that we could also have proton decay contributions due to a slightly nonzero $\lambda^{\prime \prime}$. This would open up new decay channels, for example $B+L$ conserving [56], not present in the usual Weinberg classification (although $B+L$ conserving proton decay could be mediated by $d>6$ operators even in RPC GUTs, see for example [57]). However, due to the required smallness of $\lambda^{\prime \prime}$, nothing else except baryon number violating processes would change in our analysis.

\subsection{Electroweak symmetry breaking}

Our potential is (everything is real) ${ }^{2}$

$$
V=\frac{1}{2}\left(\begin{array}{ll}
H_{u}^{0} & \tilde{\nu}_{\alpha}
\end{array}\right)\left(\begin{array}{cc}
\mu_{0}^{2}+\mu_{1}^{2}+m_{H_{u}}^{2} & -B_{\beta} \\
-B_{\alpha} & \mu_{\alpha} \mu_{\beta}+m_{\alpha \beta}^{2}
\end{array}\right)\left(\begin{array}{c}
H_{u}^{0} \\
\tilde{\nu}_{\beta}
\end{array}\right)+\frac{g^{2}+g^{\prime 2}}{32}\left(\tilde{\nu}_{\alpha}^{2}-\left(H_{u}^{0}\right)^{2}\right)^{2},
$$

where $\alpha, \beta$ run from 0 to 1 (with $m_{01}^{2}=m_{10}^{2}$ ) and we consider the basis

$$
\begin{aligned}
\left\langle H_{u}^{0}\right\rangle & =v \sin \beta, \\
\left\langle\tilde{\nu}_{0}\right\rangle & \equiv\left\langle H_{d}^{0}\right\rangle=v \cos \beta, \\
\left\langle\tilde{\nu}_{1}\right\rangle & =0 .
\end{aligned}
$$

The stationary equations give:

$$
\begin{aligned}
\mu_{0}^{2} & =\frac{m_{00}^{2}-\left(m_{H_{u}}^{2}+\mu_{1}^{2}\right) \tan ^{2} \beta}{\tan ^{2} \beta-1}-\frac{\left(g^{2}+g^{\prime 2}\right) v^{2}}{8}, \\
B_{0} & =\frac{m_{00}^{2}-\left(m_{H_{u}}^{2}+\mu_{1}^{2}\right)}{\tan ^{2} \beta-1} \tan \beta-\frac{\left(g^{2}+g^{\prime 2}\right) v^{2} \tan \beta}{4\left(\tan ^{2} \beta+1\right)}, \\
B_{1} & =\frac{\mu_{0} \mu_{1}+m_{01}^{2}}{\tan \beta} .
\end{aligned}
$$

This correctly reproduces the RPC case $\left(m_{00}=m_{H_{d}}, \mu_{1}=0, B_{1}=0\right.$ and $\left.m_{01}^{2}=0\right)$. Notice that due to $(3.22)$ we cannot take both $B_{1}$ and $m_{01}^{2}$ vanishing. This was the motivation for the assumptions (3.2) and (3.3).

By expanding $H_{u, d}^{0}=v_{u, d}+h_{u, d}^{0}$, the mass matrix of the neutral (real) scalars in the $\left(h_{u}^{0}, h_{d}^{0}, \tilde{\nu}_{1}\right)$ basis is found to be

$$
\mathcal{M}_{S}^{2}=\left(\begin{array}{ccc}
\left(m_{00}^{2}-\left(m_{H_{u}}^{2}+\mu_{1}^{2}\right)\right) \frac{1}{1-\tan ^{2} \beta}\left(m_{00}^{2}-\left(m_{H_{u}}^{2}+\mu_{1}^{2}\right)\right) \frac{-\tan \beta}{1-\tan ^{2} \beta} & \frac{m_{01}^{2}+\mu_{0} \mu_{1}}{\tan \beta} \\
\left(m_{00}^{2}-\left(m_{H_{u}}^{2}+\mu_{1}^{2}\right)\right) \frac{-\tan ^{2} \beta}{1-\tan ^{2} \beta} & \left(m_{00}^{2}-\left(m_{H_{u}}^{2}+\mu_{1}^{2}\right)\right) \frac{\tan ^{2} \beta}{1-\tan ^{2} \beta} & -\mu_{0} \mu_{1}-m_{01}^{2} \\
\frac{m_{01}^{2}+\mu_{0} \mu_{1}}{\tan \beta} & -\mu_{0} \mu_{1}-m_{01}^{2} & -\mu_{1}^{2}-m_{11}^{2}
\end{array}\right)
$$

where we also substituted the stationary conditions in eqs. (3.20)-(3.22) and we neglected $\mathcal{O}\left(v^{2}\right)$ terms. It is easy to see then, that the lightest eigenvalue (massless in the $v \rightarrow 0$ limit) is associated with the eigenvector $(\tan \beta, 1,0)$. Hence, in the decoupling limit the light Higgs has no projections on the sneutrino direction. In the finite $v$ case the component of the light Higgs in the sneutrino direction is thus proportional to $v^{2} / m_{\text {susy }}^{2}$.

\footnotetext{
${ }^{2}$ Analogously to the RPC case, the tree-level (color- and charge-preserving) minimum of the MSSM with $\mathrm{RPV}$ terms does not lead to spontaneous CP violation [58].
} 


\subsection{Neutrino masses}

In this section we will see which constraints must be satisfied in order for neutrino masses to be in the right ballpark. In doing this, we will use the mass insertion approximation for the RPV bilinear couplings as e.g. in $[35,36]$. Although this is unjustified in the present context due to large RPV couplings, we assume that they give the right order of magnitude. The purpose of this calculation is not that of predicting neutrino masses but rather to check their consistency with experimental data. In particular, we will estimate (in order of importance): the tree-level seesaw contribution from RPV interactions, the leading one-loop RPV corrections and the type I + III seesaw contribution from GUTscale mediators. Let us now discuss in turn the various cases.

\subsubsection{Tree-level seesaw from RPV interactions}

This is the most important contribution. By neglecting the typically much smaller GUTscale induced type I + III seesaw contribution (to be discuss in section 3.3.3), the only non-vanishing element of the neutrino mass matrix is

$$
m_{11}=-\frac{\mu_{1}^{2} v^{2} \cos ^{2} \beta}{4\left(\mu_{0}^{2}+\mu_{1}^{2}\right)}\left(\frac{g^{\prime 2}}{M_{1}}+\frac{g^{2}}{M_{2}}\right),
$$

where we expanded in $v / M_{1,2}$, while keeping $\mu_{1} / \mu_{0}$ of order one [37] (see also appendix A). Eq. (3.24) can be made small, for our choice of parameters, only assuming a very strong cancellation

$$
\cos ^{2} \beta\left(\frac{g^{\prime 2}}{M_{1}}+\frac{g^{2}}{M_{2}}\right) \lesssim 10^{-13} \mathrm{GeV}^{-1},
$$

i.e. having gaugino masses with opposite sign and fine-tuned ratio. This is possible since we did not assume any specific boundary condition on the soft terms (e.g. gaugino masses unification). In section 4 we will shortly comment on possible mechanisms of susy breaking which might yield to relations close to eq. (3.25).

Notice that the combination of gaugino masses in eq. (3.25) is proportional to the photino mass parameter, $m_{\tilde{\gamma}}=M_{1} c_{W}^{2}+M_{2} s_{W}^{2}$, and that the exact determinant of the generalized neutralino mass matrix in eq. (2.13) (after restricting to the nontrivial rank-5 subspace and for $\eta_{\alpha}=0$ ) is still proportional to $m_{\tilde{\gamma}}$. Though $m_{\tilde{\gamma}} \rightarrow 0$ can be effectively used to suppress large tree-level neutrino masses, this limit does not seem to be associated with any new symmetry of the Lagrangian. In fact, already at one loop this fine-tuning is not enough anymore, since the rank of the neutrino mass matrix will change as we will see in the next subsection.

\subsubsection{One-loop contributions from RPV couplings}

The most relevant diagrams for the RPV one-loop corrections to the neutrino mass matrix $[35,36,59-62]$ are shown in figure 1 . Let us now estimate their size.

i) A standard computation gives

$$
\delta m_{i j} \approx \frac{3}{16 \pi^{2}} \sum_{k, l} \lambda_{k i l}^{\prime} \lambda_{l j k}^{\prime} \frac{m_{d_{k}} m_{d_{l}}}{\tilde{m}_{d_{k}}^{2}}(A-\mu \tan \beta) .
$$



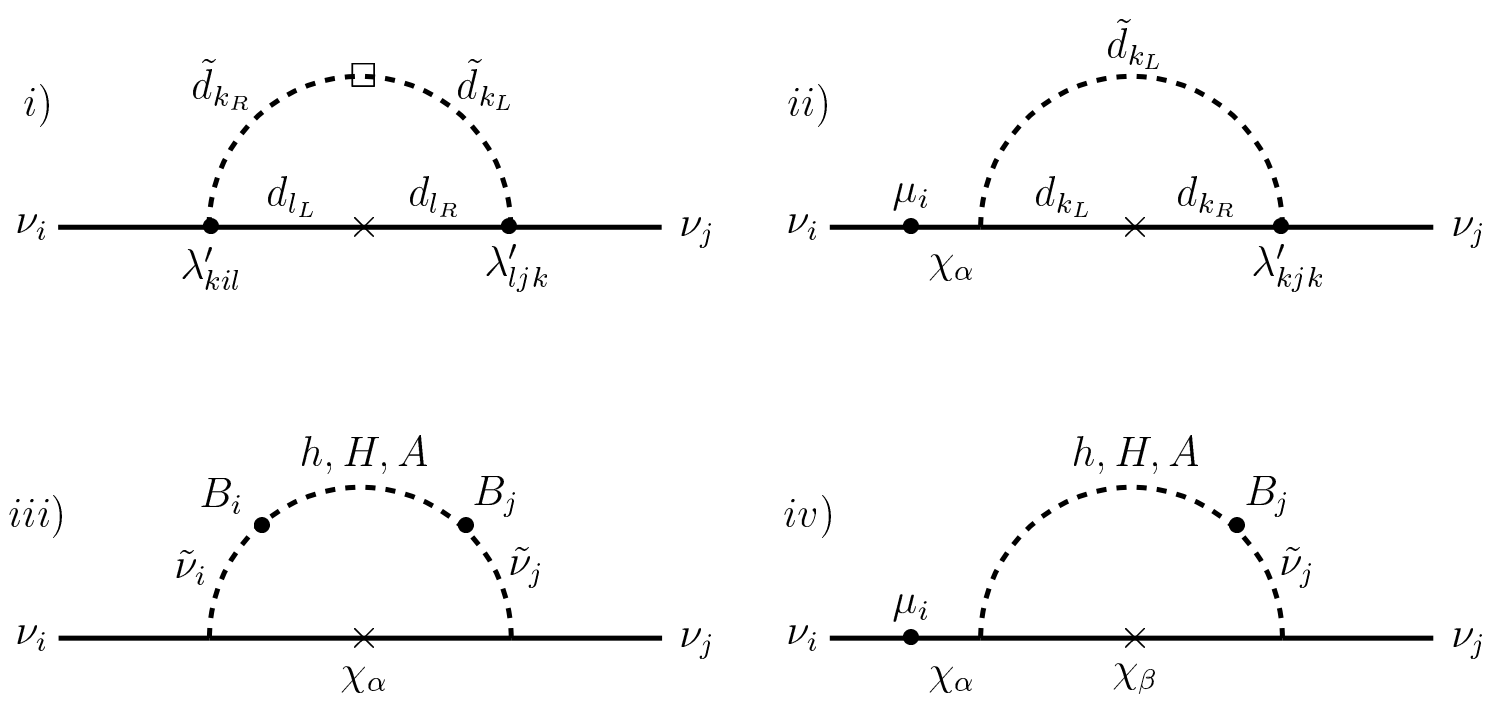

Figure 1. The dominant one-loop contributions to the neutrino mass matrix. The white square denotes a LR insertion in the squark mass matrix, while the cross stands for a mass insertion on the internal quark or neutralino propagator. The blob is associated with the source of R-parity violation.

Taking $\tan \beta=10, \mu=-1 \mathrm{TeV}, A=10 \mathrm{TeV}, \tilde{m}_{d_{i}}=30 \mathrm{TeV}, m_{d_{3}}=4.2 \mathrm{GeV}$ and the fitted values of $\lambda^{\prime}$ in eqs. (2.60)-(2.62), we get the elements of the lower right $2 \times 2$ block of the order of $100 \mathrm{eV}$, definitely too large. One can suppress these contributions by another cancellation between $A$ and $\mu \tan \beta$ and/or by increasing the sfermion masses. Similar contributions come also from two $\lambda$ s without the color factor and with sleptons running in the loop.

ii) Here the diagrams include the external neutrino mixing with both bino and wino through Higgsino; after summing all contributions and choosing a renormalization scheme such that the wino-neutrino mixing is canceled at the one-loop level [36], one gets various contributions each of the order of

$$
\delta m_{i j} \approx \frac{3}{16 \pi^{2}} \frac{g^{\prime 2} v \cos \beta}{2 \mu M_{2}} \sum_{k} m_{d_{k}}\left(\mu_{i} \lambda_{k j k}^{\prime}+\mu_{j} \lambda_{k i k}^{\prime}\right) .
$$

A more detailed calculation [36] gives an exact cancellation in the degenerate down squark case $\left(\tilde{m}_{d_{L}}^{2}=\tilde{m}_{d_{R}}^{2}\right)$. Similar diagrams with $\lambda^{\prime} \rightarrow \lambda$ and sleptons in the loop require degenerate sleptons $\left(\tilde{m}_{\tau_{L}}^{2}=\tilde{m}_{\tau_{R}}^{2}\right)$ for an exact cancellation.

iii) $+i v)$ These contributions can be written as [35, 61]

$$
\delta m_{11} \approx \frac{g^{2}}{64 \pi^{2} \cos ^{2} \beta} \frac{B_{1}^{2} m_{W}^{2}}{m_{\tilde{f}}^{6}} M_{2}+\frac{g^{2}}{64 \pi^{2} \cos \beta} \frac{B_{1} \mu_{1} m_{W}^{2}}{m_{\tilde{f}}^{4}},
$$

where we assumed $m_{W} \ll M_{2} \ll m_{\tilde{f}} \approx m_{H_{u, d}}$. Notice the $m_{W}^{2} / m_{\tilde{f}}^{2}$ suppression in eq. (3.28), which is a remnant of an exact cancellation of the loop functions in the decoupling limit [61]. These contributions are in the same direction as the fine-tuned tree-level one. So all one needs is doing just a slightly different fine-tuning. 


\subsubsection{Seesaw from GUT-scale mediators}

For completeness, we estimate the rank-1 type I + III seesaw contribution from GUT-scale mediators in eq. (2.13) in the limit of no RPV mixing. This yields one non-vanishing neutrino mass eigenvalue

$$
m_{\nu}=\eta^{2} v_{u}^{2} / M_{\text {seesaw }}
$$

where $\eta=\sqrt{\eta_{0}^{2}+\eta_{k}^{2}}$ and $\eta=\mathcal{O}(1)$ in order to achieve the doublet-triplet splitting (cf. section 2). Notice that, since $M_{\text {seesaw }}$ could be as large as $M_{\mathrm{GUT}} \gtrsim 10^{16} \mathrm{GeV}$, this contribution to neutrino masses can be made subleading.

In conclusion, neutrino masses can be (admittedly barely) under control assuming a strong fine-tuning among wino and bino mass parameters (3.25) to suppress the tree-level contribution, heavy sfermions or small left-right sfermion mixings to suppress (3.26), an approximate degeneracy in the sfermion spectrum to suppress the one-loop contribution (3.27) and $M_{\text {seesaw }} \approx M_{\mathrm{GUT}}$.

\subsection{Modifications of SM couplings to leptons}

The mixing between leptons and higgsinos/gauginos is also constrained by the measurement of the SM couplings to the lightest lepton mass eigenstates $\hat{e}_{1,2,3}$ and $\hat{\nu}_{1,2,3}$. The relevant couplings to be considered here are: $Z \hat{e}_{i} \hat{e}_{j}$ (precision measurement at the $Z$ pole and lepton flavour violating charged lepton decays), $Z \hat{\nu}_{i} \hat{\nu}_{j}$ (invisible $Z$ width) and $W \hat{e}_{i} \hat{\nu}_{j}$ (charged lepton universality).

Assuming real parameters and denoting the deviation from a SM coupling $g_{\mathrm{SM}}$ as $\delta g_{\mathrm{SM}}$, the modified SM couplings to leptons are found to be (see also [37, 63, 64]):

- $Z \hat{e}_{i} \hat{e}_{j}$ couplings:

$$
\begin{aligned}
\delta g_{L}^{i j} & =U_{L}^{i+2,1} U_{L}^{j+2,1}, \\
\delta g_{R}^{i j} & =2 U_{R}^{i+2,1} U_{R}^{j+2,1}+U_{R}^{i+2,2} U_{R}^{j+2,2},
\end{aligned}
$$

where $U_{L, R}$ are the bi-unitary matrices which diagonalize the generalized chargino mass matrix (cf. appendix A), while $i$ and $j$ run over the three lightest eigenvalues. In particular, in the susy-decoupling limit considered in appendix A we get:

$$
\begin{aligned}
\left|U_{L}^{31}\right| & =\left|\frac{g v_{d} \mu_{1}}{\sqrt{2} \mu M_{2}}\right|=\mathcal{O}\left(m_{W} / M_{2}\right), \\
\left|U_{R}^{31}\right| & =\left|\frac{\mu_{1} m_{1}}{\mu^{2}} \frac{g v_{u}}{\sqrt{2} M_{2}}\right|=\mathcal{O}\left(m_{1} m_{W} /\left(\mu M_{2}\right)\right), \\
\left|U_{R}^{32}\right| & =\left|\frac{\mu_{1} m_{1}}{\mu^{2}}\right|=\mathcal{O}\left(m_{1} / \mu\right),
\end{aligned}
$$

and the modified couplings of the $Z$ boson to charged leptons (electrons) are hence $\delta g_{L}^{11}=\mathcal{O}\left(m_{W}^{2} / M_{2}^{2}\right)$ and $\delta g_{R}^{11}=\mathcal{O}\left(m_{1}^{2} / \mu^{2}\right)$.

The constraints from the $Z$-pole observables are typically given in terms of $\delta g_{V, A}=$ $\frac{1}{2}\left(\delta g_{L} \pm \delta g_{R}\right)$ and are at most at the $0.07 \%$ level for the flavour diagonal case [65-67]. 
On the other hand, the bounds on the flavour violating couplings are less strict, with the only exception of those coming from the measurement of $\mu \rightarrow e e e^{c}$, which sets $\delta g_{V, A}^{12} \lesssim 10^{-6}[63,68]$. The latter bound is evaded by our specific flavour orientation of the $\mu_{i}$ vector, e.g. $\mu_{i} \propto \delta_{1 i}$.

Hence, all the relevant bounds due to the modification of the $Z$ boson couplings to charged leptons are satisfied by $M_{2} \gtrsim 5 \mathrm{TeV}$ and $\mu_{i} \propto \delta_{1 i}$.

- $Z \hat{\nu}_{i} \hat{\nu}_{j}$ couplings:

$$
\delta g_{V, A}^{i j}=-U_{0}^{i+4,1} U_{0}^{j+4,1}-U_{0}^{i+4,2} U_{0}^{j+4,2}-2 U_{0}^{i+4,3} U_{0}^{j+4,3},
$$

where $U_{0}$ is the unitary matrix which diagonalizes the generalized neutralino mass matrix (cf. appendix A), while $i$ and $j$ run over the three lightest eigenvalues.

At the leading order in the expansion of appendix A, we find

$$
\begin{aligned}
& \left|U_{0}^{51}\right|=\left|\frac{g^{\prime} v_{d} \mu_{1}}{2 \mu M_{1}}\right|=\mathcal{O}\left(m_{W} / M_{1}\right), \\
& \left|U_{0}^{52}\right|=\left|\frac{g v_{d} \mu_{1}}{2 \mu M_{2}}\right|=\mathcal{O}\left(m_{W} / M_{2}\right) .
\end{aligned}
$$

For $\mu>m_{Z}$, the typical signature is the reduction of the invisible width of the $Z$ boson. However, even for moderate (non-decoupled) values of $M_{1,2}$, the inferred bound on $\mu_{1}$ is very mild [63].

- $W \hat{e}_{i} \hat{\nu}_{j}$ couplings: defining the current eigenstate matrices

$$
\begin{aligned}
T^{L} & =\left(\begin{array}{cccc}
0 & \sqrt{2} & 0 & 0 \\
0 & 0 & 0 & 1_{4 \times 4}
\end{array}\right), \\
T^{R} & =\left(\begin{array}{cccc}
0 & -\sqrt{2} & 0 & 0 \\
0 & 0 & 1 & 0 \\
0 \times 4
\end{array}\right),
\end{aligned}
$$

the modified SM couplings read

$$
\begin{aligned}
& \delta \tilde{g}_{L}^{i j}=\left(U_{L}^{\dagger} T^{L} U_{0}\right)^{i j}, \\
& \delta \tilde{g}_{R}^{i j}=\left(U_{R}^{\dagger} T^{R} U_{0}\right)^{i j},
\end{aligned}
$$

where $i$ and $j$ run over the three lightest eigenvalues. Charged lepton universality in charged current processes, such as the decay of pions and leptons, is experimentally verified at the $0.2 \%$ level [69]. This typically yields less stringent bounds than those derived from $Z$ couplings [63].

Summarizing, the couplings of the $Z$ and $W$ bosons to the three lightest lepton mass eigenstates can be easily made compatible with the SM values by a moderate decoupling of gaugino masses (say $M_{1,2} \gtrsim 5 \mathrm{TeV}$ ) and for $\mu_{i} \propto \delta_{i 1}$. This was indeed to be expected, since in the gaugino decoupling limit we are mixing only representations with the same gauge quantum numbers (GIM-like mechanism), and hence gauge couplings have to be SM-like. 


\subsection{Other lepton number violating processes}

On top of neutrino masses there are also other lepton number violating effects which are worth to be discussed. First of all, LHC can produce via a Drell-Yan process a pair of winos which can subsequently decay through lepton number violating couplings into same-sign dileptons [70] and 4 jets with no missing energy (ideally, a background-free process):

$$
p p \rightarrow W^{* \pm} \rightarrow \tilde{W}^{ \pm} \tilde{W}^{0} \rightarrow\left(e^{ \pm} Z\right)\left(e^{ \pm} W^{\mp}\right) \rightarrow\left(e^{ \pm} j j\right)\left(e^{ \pm} j j\right) .
$$

This is completely analogous the the production and decay of a light weak triplet fermion pair from type III seesaw [71-74]. Since winos are unstable the cross section $\sigma\left(p p \rightarrow \tilde{W}^{ \pm} \tilde{W}^{0}\right)$ gets multiplied with an approximate factor

$$
\int_{E_{\min }^{2}}^{E_{\max }^{2}} \frac{M_{2} \Gamma d p^{2}}{\left(p^{2}-M_{2}^{2}\right)^{2}+M_{2}^{2} \Gamma_{\text {TOT }}^{2}},
$$

for each wino. For $E_{\min }^{2} \ll M_{2}^{2} \ll E_{\max }^{2}$ the integral can be approximated by the branching fraction of the decay channel. This is what happens in the usual MSSM with light $M_{2}$ and small RPV couplings.

However, since in our case winos are typically much heavier than the electroweak scale $\left(M_{2} \gtrsim 5 \mathrm{TeV}\right.$ from the modified $Z$ couplings - see section 3.4), we should replace (very roughly)

$$
\begin{aligned}
& B R\left(\tilde{W}^{ \pm} \rightarrow e^{ \pm} Z\right) B R\left(\tilde{W}^{0} \rightarrow e^{ \pm} W^{\mp}\right) \longrightarrow \\
&\left(\frac{E_{\max }}{M_{2}}\right)^{4}\left(\frac{\Gamma\left(\tilde{W}^{ \pm} \rightarrow e^{ \pm} Z\right)}{M_{2}}\right)\left(\frac{\Gamma\left(\tilde{W}^{0} \rightarrow e^{ \pm} W^{\mp}\right)}{M_{2}}\right) .
\end{aligned}
$$

This is small due to the $\left(m_{W} / M_{2}\right)^{2}$ suppression of the $\Gamma$ (see eq. (A.15)) and eventually because $E_{\max }<M_{2}$. Hence, in spite of the fact that the RPV coupling $\mu_{1} / \mu_{0}$ is much larger than in the usual case, this lepton number violating process will not be easily accessible at LHC because the ratio $m_{W} / M_{2} \lesssim 1 / 50$ is too small, giving for eq. (3.44) a suppression of $\approx 10^{-7}$.

The next lepton number violating process we consider is neutrinoless double $\beta$ decay. Following [14] the limits on the trilinear RPV couplings are $(k=1,2,3)$

$$
\begin{aligned}
\left|\lambda_{111}^{\prime}\right|^{2}\left(\frac{m_{W}}{m_{\tilde{f}}}\right)^{4}\left(\frac{m_{W}}{m_{\lambda}}\right) & \lesssim 10^{-8}, \\
\left|\lambda_{11 k}^{\prime} \lambda_{k 11}^{\prime}\right|\left(\frac{m_{W}}{m_{\tilde{f}}}\right)^{4}\left(\frac{A-\mu \tan \beta}{m_{W}}\right) & \lesssim 10^{-(6 \div 8),}
\end{aligned}
$$

which are easily satisfied in our case, even for relatively low super-partner masses. On the other hand, the parameter $\mu_{1} / \mu_{0}$ contributes to the process only through the light neutrino masses, whose suppression has been already discussed in section 3.3.

Finally, other potentially relevant lepton number violating processes like e.g. $\mu^{+} \rightarrow e^{-}$ conversion in nuclei, $K^{+} \rightarrow \mu^{+} \mu^{+} \pi^{-}$or $\bar{\nu}_{e}$ emission from the Sun, do not bring any really important constraint on the model parameters since the experimental limits on the branching ratios are still too weak. 


\subsection{Lepton flavour violation}

In this section we analyse in more detail lepton flavour violating processes like $\mu \rightarrow e$ conversion in nuclei, $\mu \rightarrow e e e^{c}$ and $\mu \rightarrow e \gamma$ (other processes involving the $\tau$ lepton are worse measured and their bounds can be easily evaded). At leading order $\left(\epsilon^{0}\right)$ in $\epsilon=$ $\mathcal{O}\left(m_{W} / M_{2}, m_{1} / m_{W}\right) \lesssim 10^{-2}$ there is no mixing between generations, i.e. the electron mass eigenstate mixes just with Higgsino, while the muon does not mix at all $\left(\mu_{2}=0\right)$, see appendix A. In other words, at order $\epsilon^{0}$ and tree level the $\lambda$ and $\lambda^{\prime}$ couplings are already in the mass eigenbasis. In particular, all the lepton flavour changing amplitudes involving electrons vanish at order $\epsilon^{0}$. Following for example the computation and notation of [75] for $\mu \rightarrow e$ conversion and [76] for the other two processes, we can summarize the results as follows ( $\lambda$ and $\lambda^{\prime}$ corresponding to the values determined in section 2.6):

- $\mu \rightarrow e$ conversion: the coefficients in front of the possible operators of the type $\bar{e} \mu \bar{q} q$ are at tree order

$$
\begin{gathered}
A^{d} \sim+\sum_{k=1}^{3} \frac{\lambda_{11 k}^{\prime} \lambda_{12 k}^{\prime}}{m_{\tilde{Q}_{k}}^{2}} \rightarrow 0, \quad A^{u} \sim-\sum_{k=1}^{3} \frac{\lambda_{k 11}^{\prime} \lambda_{k 21}^{\prime}}{m_{\tilde{d}_{k}}^{2}} \rightarrow 0, \\
S^{d, 1} \sim-2 \sum_{k=1}^{3} \frac{\lambda_{1 k 1}^{\prime} \lambda_{k 12}}{m_{\tilde{Q}_{k}}^{2}} \rightarrow 0, \quad S^{d, 2} \sim-2 \sum_{k=1}^{3} \frac{\lambda_{1 k 1}^{\prime} \lambda_{k 21}}{m_{\tilde{Q}_{k}}^{2}} \rightarrow 0 .
\end{gathered}
$$

- $\mu \rightarrow e e e^{c}$ : the coefficients in front of the possible operators of the type $\bar{e} \mu \bar{e} e$ are at tree order

$$
B^{L} \sim-\sum_{k=1}^{3} \frac{\lambda_{k 11} \lambda_{k 21}}{2 m_{\tilde{L}_{k}}^{2}} \rightarrow 0, \quad B^{R} \sim-\sum_{k=1}^{3} \frac{\lambda_{k 11} \lambda_{k 12}}{2 m_{\tilde{L}_{k}}^{2}} \rightarrow 0 .
$$

- $\mu \rightarrow e \gamma$ : the coefficients in front of the possible operators are at one-loop order

$$
\begin{aligned}
& A_{2}^{R} \sim \frac{1}{16 \pi^{2}} \frac{1}{12} \sum_{j, k=1}^{3}\left(-2 \frac{\lambda_{1 k j} \lambda_{2 k j}}{m_{\tilde{L}_{k}}^{2}}+\frac{\lambda_{1 k j} \lambda_{2 k j}}{m_{\tilde{e}_{k}}^{2}}-3 \frac{\lambda_{k 1 j}^{\prime} \lambda_{k 2 j}^{\prime}}{m_{\tilde{d}_{k}}^{2}}\right) \rightarrow 0, \\
& A_{2}^{L} \sim \frac{1}{16 \pi^{2}} \frac{1}{12} \sum_{j, k=1}^{3}\left(-2 \frac{\lambda_{k j 1} \lambda_{k j 2}}{m_{\tilde{L}_{k}}^{2}}+\frac{\lambda_{j k 1} \lambda_{j k 2}}{m_{\tilde{L}_{k}}^{2}}\right) \rightarrow 0 .
\end{aligned}
$$

Next we want to check what happens beyond the leading order. Without doing a full calculation for the order $\epsilon$ or at higher loops, we can consider the following:

1. Either $\epsilon$ or an extra loop factor contribute with a suppression factor of at least $10^{-2}$;

2. Although $L_{1}$ violation is in principle order one, $L_{2}$ violation is of order $10^{-1}$ (cf. discussion below eq. (2.62));

3. The propagator gets a suppression $\left(m_{W} / m_{\tilde{f}}\right)^{2}$ compared to the Fermi constant $G_{F}$

Putting all this together, we schematically find for the generic coefficient $\mathcal{A}$ in eqs. (3.47)(3.51) relative to the different processes: 
- $\mu \rightarrow e$ conversion: comparing theoretical expectations [77] with the experimental constraint on Titanium [78]

$$
m_{W}^{2} \mathcal{A}_{\mu-e} \sim 10^{-2} 10^{-1}\left(\frac{m_{W}}{m_{\tilde{f}}}\right)^{2} \lesssim 10^{-7},
$$

which can be satisfied for sfermion masses of order $10 \mathrm{TeV}$ or more.

- $\mu \rightarrow e e e^{c}$ : similar estimates give (see [67] for experimental bounds)

$$
m_{W}^{2} \mathcal{A}_{\mu \rightarrow 3 e} \sim 10^{-2} 10^{-1}\left(\frac{m_{W}}{m_{\tilde{f}}}\right)^{2} \lesssim 10^{-6},
$$

again easily satisfied for $\tilde{m}_{f} \gtrsim 3 \mathrm{TeV}$.

- $\mu \rightarrow e \gamma$ : following again [67] we find (notice that here we started already at one-loop)

$$
m_{W}^{2} \mathcal{A}_{\mu \rightarrow e \gamma} \sim\left(10^{-2}\right)^{2} 10^{-1}\left(\frac{m_{W}}{m_{\tilde{f}}}\right)^{2} \lesssim 10^{-6},
$$

which is evaded already for $m_{\tilde{f}} \gtrsim 300 \mathrm{GeV}$.

\subsection{Gravitino dark matter}

In the presence of sizeable RPV interactions there are no long-lived states in the MSSM spectrum, so the only DM candidate is a slowly decaying gravitino. For $m_{3 / 2}<m_{Z}$ the main decay channel of the gravitino is [80]

$$
\Gamma(\tilde{G} \rightarrow \gamma \nu)=\frac{1}{32 \pi}\left|U_{\tilde{\gamma} \nu}\right|^{2} \frac{m_{3 / 2}^{3}}{M_{P}^{2}},
$$

where $U_{\tilde{\gamma} \nu}=c_{W} U_{\tilde{B} \nu}+s_{W} U_{\tilde{W} \nu}$ is the photino-neutrino mixing and $M_{P}=2.4 \times 10^{18} \mathrm{GeV}$ is the reduced Planck mass. From eq. (A.27) we read

$$
\left|U_{\tilde{\gamma} \nu}\right|^{2}=\frac{\pi \alpha_{\mathrm{em}} v_{d}^{2} \mu_{1}^{2}}{\mu^{2}}\left(\frac{1}{M_{1}}-\frac{1}{M_{2}}\right)^{2} \approx 10^{-7}\left(\frac{10}{\tan \beta}\right)^{2}\left(\frac{10 \mathrm{TeV}}{M_{1}}\right)^{2},
$$

where $\tan \beta \gg 1$ and we already considered the fine-tuning in eq. (3.25) in order to suppress neutrino masses. This has to be compared with the standard case where the smallness of neutrino masses is due to a tiny mixing with gauginos, yielding [80]

$$
\left|U_{\tilde{\gamma} \nu}\right|_{\text {stand }}^{2}=\mathcal{O}\left(\frac{m_{\nu}}{M_{1}}\right)
$$

or, equivalently

$$
\left|U_{\tilde{\gamma} \nu}\right|^{2} /\left|U_{\tilde{\gamma} \nu}\right|_{\text {stand }}^{2} \approx 10^{6}\left(\frac{10}{\tan \beta}\right)^{2}\left(\frac{10 \mathrm{TeV}}{M_{1}}\right)
$$

Hence, in our scenario, where neutrino masses and $U_{\tilde{\gamma} \nu}$ mixing are decoupled, the gravitino decays a factor $\approx 10^{6}$ faster than in the standard RPV case and so we have to check whether it can still be a good DM candidate. 
As a first check let us compare its lifetime with the age of the Universe $\tau_{U} \approx 4.3 \times 10^{17}$ s. From eqs. (3.55)-(3.56) we obtain

$$
\tau_{3 / 2} \approx 3.8 \times 10^{18} \mathrm{~s}\left(\frac{\tan \beta}{10}\right)^{2}\left(\frac{M_{1}}{10 \mathrm{TeV}}\right)^{2}\left(\frac{10 \mathrm{GeV}}{m_{3 / 2}}\right)^{3},
$$

which is safe, as long as $m_{3 / 2} \lesssim 10 \mathrm{GeV}$ (for $M_{1} \approx 10 \mathrm{TeV}$ and $\tan \beta \approx 10$ ).

The decay of the gravitino is expected to leave an imprint on the extragalactic diffuse high-energy photon background in the form of a monochromatic line centred at $m_{3 / 2} / 2$. This is because $m_{3 / 2}$ is very light, contrary to what happens with multi-TeV gravitino masses where a continuum signal in the spectrum is expected, see for example [79]. The photon number flux, $F_{\gamma}^{\max }$, at the peak of the maximum photon energy $E_{\gamma}=m_{3 / 2} / 2$, is estimated to be [80]

$$
F_{\gamma}^{\max } \approx 10^{-5} \mathrm{~cm}^{-2} \mathrm{sr}^{-1} \mathrm{~s}^{-1}\left(\frac{m_{3 / 2}}{10 \mathrm{MeV}}\right)^{2}\left(\frac{\Omega_{3 / 2} h^{2}}{0.12}\right)\left(\frac{10}{\tan \beta}\right)^{2}\left(\frac{10 \mathrm{TeV}}{M_{1}}\right)^{2},
$$

which is compatible with the bounds coming from diffuse $\mathrm{X}$ - and gamma-ray fluxes (see figure 1 in [81], [82, 83]), as long as $m_{3 / 2} \lesssim 10 \mathrm{MeV}$ (for $M_{1} \approx 10 \mathrm{TeV}$ and $\tan \beta \approx 10$ ). The latter values correspond to a lifetime $\tau_{3 / 2}>10^{27 \div 28} \mathrm{~s}$, which is indeed the typically constraint for decaying DM into photons [84]. Notice, also, that there are no observational constraints (from Big Bang Nucleosynthesis or CMB) on the decay of the next-to-lightest supersymmetric particle, due to its fast decay via large RPV interaction.

The last point we want to address is a possible constraint related to the reheating temperature. Assuming thermal production in the early Universe, the gravitino relic density is constrained by (see e.g. [85-88])

$$
\Omega_{3 / 2} h^{2} \gtrsim 0.12\left(\frac{T_{R H}}{300 \mathrm{GeV}}\right)\left(\frac{10 \mathrm{MeV}}{m_{3 / 2}}\right)\left(\frac{M_{2}}{30 \mathrm{TeV}}\right)^{2},
$$

where approximate equality holds when the gluino contribution can be neglected. Notice that for $m_{3 / 2} \lesssim 10 \mathrm{MeV}$ and $M_{2} \approx 30 \mathrm{TeV}\left(M_{1} \approx-M_{2} g^{\prime 2} / g^{2} \approx 9 \mathrm{TeV}\right)$, the reheating temperature can still be above the electroweak phase transition. On the other hand, gravitino masses lighter than already $1 \mathrm{MeV}$ (or, equivalently, too large gaugino masses) would imply a reheating temperature well below the electroweak phase transition, which is difficult to reconcile with an high-energy mechanism of baryogenesis. ${ }^{3}$ From this point of view, a gravitino mass close to the upper limit of $10 \mathrm{MeV}$ (compatible with the measured photon fluxes) is theoretically favourable. This is, of course, also the most interesting region for a possible experimental discovery.

\section{Discussion and conclusions}

Among grand unified theories only renormalizable $\mathrm{SO}(10)$ [89-91] is able to derive exact R-parity conservation [92-94] at low energies [95-97], while there is no reason to assume

\footnotetext{
${ }^{3}$ In our setup baryon number is effectively preserved below the GUT scale, therefore we are only left with the possibility of generating a lepton asymmetry above the electroweak phase transition and get it converted into a baryon one through sphalerons effects.
} 
it in $\mathrm{SU}(5)$. There are of course strong phenomenological constraints that make especially the baryon number violating couplings practically zero. In this work we tried to see if the remaining R-parity violating interactions in the minimal renormalizable $\mathrm{SU}(5)$ can be of any utility for the down quark vs. charged lepton mass problem of the original setup. The outcome of our analysis is positive: these couplings are able to reproduce the SM fermion masses and so avoid large susy breaking threshold corrections which would make our vacuum metastable [13]. The prize to pay are three classes of fine-tuning: i) a generalized doublet-triplet splitting (cf. eqs. (2.9)-(2.10)), ii) the vanishing of the baryonic RPV couplings $\lambda^{\prime \prime}$ in eq. (2.53) and iii) the suppression of neutrino masses in eq. (3.25).

Is relation (3.25) between gaugino masses a prediction of the theory? Since it gets corrections at higher loops, eq. (3.28) being the dominant one, the question is thus: how exactly must $M_{1} / g^{\prime 2}=-M_{2} / g^{2}$ hold? Let us see what we need for this relation to be for example $10 \%$ exact, i.e. suppose

$$
\frac{M_{1}}{g^{\prime 2}}=-\frac{M_{2}}{g^{2}}(1 \pm 0.1)
$$

This is equivalent to say that the loop contribution is at most $10 \%$ of the non-fine-tuned value in eq. (3.24), i.e.

$$
\delta m_{11} \lesssim \frac{1}{10} \times \frac{\mu_{1}^{2} v^{2} \cos ^{2} \beta}{4\left(\mu_{0}^{2}+\mu_{1}^{2}\right)} \frac{g^{2}}{M_{2}} .
$$

In usual perturbation theory $\delta m / m$ is loop suppressed, so small, provided the same couplings as at tree order are used. But in our case we have more like a Coleman-Weinberg situation [98], where new couplings not present at tree level, in our case $B_{1}$, start contributing. So there is no limitation from perturbation theory and at least in principle loops could dominate over tree-level contributions. Is this what happens here? According to (3.28), and assuming a split susy spectrum $\mu_{1} \sim M_{2} \ll m_{\tilde{f}} \sim \sqrt{\left|B_{1}\right|}$ we find that very roughly the $10 \%$ correlation between bino and wino mass (4.1) is valid if

$$
M_{2} \lesssim 10 \cos ^{2} \beta m_{\tilde{f}} .
$$

For larger $M_{2}$, there is still a strong correlation between $M_{1}$ and $M_{2}$, but other parameters get involved too, so it is harder to make a definite statement of what to look for. But if eq. (4.3) is valid, the apparently weak point of the neutrino mass becomes a strong one, and the theory is falsifiable through a future experimental check of eq. (4.1).

Suppose now that $M_{2}$ satisfies eq. (4.3). Is there any obvious theoretical reason why would eq. (4.1) hold? In other words, can one find a susy breaking and mediation mechanism which leads to it at least at the one-loop level? A natural candidate would be gauge mediation. The change in sign of the bino mass compared to wino mass can be obtained only by a combination of gauge messengers (which contribute negatively) with chiral messengers (which contribute positively). A naive simple computation shows that if an SU(5) adjoint breaks susy like for example in [99, 100], one needs [101]

$$
1=\frac{\left(M_{1} / g_{1}^{2}\right)}{(-3 / 5)\left(M_{2} / g_{2}^{2}\right)}=\frac{\left(\Delta b_{\text {chiral }}-10\right)}{(-3 / 5)\left(\Delta b_{\text {chiral }}-6\right)}
$$


where we assumed that chiral superfields contribute in SU(5) multiplets. The change of the $\mathrm{SU}(5)$ beta function equals $\Delta b_{\text {chiral }}=17 / 2$ on the threshold. A half-integer $\Delta b_{\text {chiral }}$ seems impossible to obtain: a complex representation needs always to come in pairs to be vectorlike and satisfy anomaly constraints, while real representations have an integer Dynkin index. Evading this conclusion needs more sophisticated scenarios. However, if (4.1) is relaxed a bit (by $M_{1,2} \gtrsim 10 m_{\tilde{f}}$ and/or large $\tan \beta$ ), then we can get with an integer $\Delta b_{\text {chiral }}$ (for example 8 or 9 ) opposite sign bino and wino masses.

Another possibility is to consider gravity mediation. From $[102]^{4}$ we see that relation (4.1) is obtained for example in $\mathrm{SO}(10)$ if a 210 is coupled to gauge field strength bilinears and its parity odd Pati-Salam singlet gets a non-zero F-term. Although amusing, it is unclear what this means in the context of our renormalizable SU(5) model.

On top of eq. (4.1), the other prediction is a gravitino dark matter candidate lighter than approximately $10 \mathrm{MeV}$, preferably closer to the upper limit in order to be reconcilable with baryogenesis. A gravitino mass in the region favoured by baryogenesis is also the most interesting one from an experimental point of view. The main signature being a monochromatic line in the diffuse extragalactic photon background picked around $5 \mathrm{MeV}$.

In this work we only used the RPV mixing effects to correct the wrong SU(5) mass relations. In practice, however, the solution to this problem could arise from different sources, partially from susy threshold corrections and partially from RPV mixings, thus modifying the numerical values of the RPV parameters here considered. Also, the ad-hoc assumption of setting to zero those couplings that make the wrong mass relations worse, is not really needed, although a generic situation might be forbidden by data.

Although the model is a bit stretched and many tunings of parameters are needed, the phenomenology itself seems interesting: the electron mass eigenstate (or other leptons as well in a more general framework) may not be what we usually think of, but rather an order half-electron and half-Higgsino flavour state.

\section{Acknowledgments}

We are deeply indebted with Sacha Davidson for several valuable discussions, clarification regarding her work and correspondence. We thank Marco Nardecchia for collaboration in the early stages of this project, Giorgio Arcadi, Ilia Gogoladze, Miha Nemevšek and Gabrijela Zaharijas for discussions, Stephane Lavignac, Goran Senjanović and Vasja Susič for reading the manuscript and useful comments. We thank Kaladi Babu for pointing out an error in a previous version of this paper. The work of B.B. has been supported by the Slovenian Research Agency. B.B. would like to thank CETUP* (Center for Theoretical Underground Physics and Related Areas), supported by the US Department of Energy under Grant No. DE-SC0010137 and by the US National Science Foundation under Grant No. PHY-1342611, for its hospitality and partial support during the 2013 Summer Program. B.B. acknowledges the hospitality and support from the NORDITA scientific program News in Neutrino Physics, April 7-May 2, 2014, during which part of this study was performed.

\footnotetext{
${ }^{4}$ We thank Ilia Gogoladze for pointing out this possibility.
} 
The work of L.D.L. is supported by the Marie Curie CIG program, project number PCIG13GA-2013-618439. L.D.L. is grateful to the theoretical phyisics group of the Jožef Stefan Institute for the warm hospitality and support during the development of this project.

\section{A Perturbative diagonalization}

Let us write the diagonalization of the generalized chargino and neutralino mass matrices, in eq. (2.15) and eq. (2.13) respectively, as:

$$
\begin{aligned}
U_{L}^{\dagger} \mathcal{M}_{C} U_{R} & =\operatorname{diag}\left(\hat{M}_{c 1}, \hat{M}_{c 2}, \hat{m}_{e 1}, \hat{m}_{e 2}, \hat{m}_{e 3}\right), \\
U_{0}^{\dagger} \mathcal{M}_{N} U_{0} & =\operatorname{diag}\left(\hat{M}_{n 1}, \hat{M}_{n 2}, \hat{M}_{n 3}, \hat{M}_{n 4}, \hat{m}_{\nu 1}, \hat{m}_{\nu 2}, \hat{m}_{\nu 3}\right) .
\end{aligned}
$$

For simplicity we will consider real parameters and limit ourselves to the case where only $\mu_{1} \neq 0\left(\mu_{2}=\mu_{3}=0\right)$. For a more general case see e.g. [63]. Then the relevant squared mass matrices in the chargino sector read

$$
\mathcal{M}_{C}^{T} \mathcal{M}_{C}=\left(\begin{array}{ccccc}
M_{2}^{2}+g^{2} v_{d}^{2} / 2 & M_{2} g v_{u} / \sqrt{2}+\mu_{0} g v_{d} / \sqrt{2} & 0 & 0 & 0 \\
M_{2} g v_{u} / \sqrt{2}+\mu_{0} g v_{d} / \sqrt{2} & \mu_{0}^{2}+\mu_{1}^{2}+g^{2} v_{u}^{2} / 2 & m_{1} \mu_{1} & 0 & 0 \\
0 & m_{1} \mu_{1} & m_{1}^{2} & 0 & 0 \\
0 & 0 & 0 & m_{2}^{2} & 0 \\
0 & 0 & 0 & 0 & m_{3}^{2}
\end{array}\right)
$$

which is diagonalized by $U_{R}$, and

$$
\mathcal{M}_{C} \mathcal{M}_{C}^{T}=\left(\begin{array}{ccccc}
M_{2}^{2}+g^{2} v_{u}^{2} / 2 & \left(M_{2} g v_{d}+\mu_{0} g v_{u}\right) / \sqrt{2} & \mu_{1} g v_{u} / \sqrt{2} & 0 & 0 \\
\left(M_{2} g v_{d}+\mu_{0} g v_{u}\right) / \sqrt{2} & \mu_{0}^{2}+g^{2} v_{d}^{2} / 2 & \mu_{0} \mu_{1} & 0 & 0 \\
\mu_{1} g v_{u} / \sqrt{2} & \mu_{0} \mu_{1} & m_{1}^{2}+\mu_{1}^{2} & 0 & 0 \\
0 & 0 & 0 & m_{2}^{2} & 0 \\
0 & 0 & 0 & 0 & m_{3}^{2}
\end{array}\right)
$$

which is relevant for the determination of $U_{L}$.

The $7 \times 7$ neutralino mass matrix is given in eq. (2.13), with $\mu_{2}=\mu_{3}=0$ and $\eta_{\alpha}=0$. We neglect the contribution of the type I + III seesaw, since it can be made subleading (cf. section 3.3.3).

Working in the phenomenological limit $M_{1,2} \approx \mu_{0,1} \gg v_{u, d}=\mathcal{O}\left(m_{W}\right) \gg m_{1}$, at the first order in the expansion parameter $\epsilon=m_{W} / M_{1,2}$ or $m_{1} / m_{W} \ll 1$ we find:

- Chargino sector:

$$
\begin{aligned}
& \hat{M}_{c 1}(\epsilon)=M_{2}, \\
& \hat{M}_{c 2}(\epsilon)=\mu, \\
& \hat{m}_{1}(\epsilon)=m_{1} \sqrt{1-\frac{\mu_{1}^{2}}{\mu^{2}}}, \\
& \hat{m}_{2}(\epsilon)=m_{2}, \\
& \hat{m}_{3}(\epsilon)=m_{3},
\end{aligned}
$$


where $\mu=\sqrt{\mu_{0}^{2}+\mu_{1}^{2}}$. The perturbed eigenvectors (normalized up to $\mathcal{O}\left(\epsilon^{2}\right)$ corrections) read

$$
\begin{aligned}
\tilde{W}^{+}(\epsilon) & =\tilde{W}^{+}+\frac{g v_{u}}{\sqrt{2} M_{2}} \tilde{H}_{u}^{+}, \\
\tilde{H}_{u}^{+}(\epsilon) & =-\frac{g v_{u}}{\sqrt{2} M_{2}} \tilde{W}^{+}+\tilde{H}_{u}^{+}+\frac{\mu_{1} m_{1}}{\mu^{2}} e_{1}^{c}, \\
e_{1}^{c}(\epsilon) & =-\frac{\mu_{1} m_{1}}{\mu^{2}} \tilde{H}_{u}^{+}+e_{1}^{c}, \\
e_{2}^{c}(\epsilon) & =e_{2}^{c} \\
e_{3}^{c}(\epsilon) & =e_{3}^{c}
\end{aligned}
$$

and

$$
\begin{aligned}
\tilde{W}^{-}(\epsilon) & =\tilde{W}^{-}+\frac{g v_{d}}{\sqrt{2} M_{2}} \tilde{H}_{d}^{-} \\
\tilde{H}_{d}^{-}(\epsilon) & =-\frac{g v_{d}}{\sqrt{2} M_{2}} \tilde{W}^{-}+\frac{\mu_{0}}{\mu} \tilde{H}_{d}^{-}+\frac{\mu_{1}}{\mu} e_{1}, \\
e_{1}(\epsilon) & =\frac{g v_{d} \mu_{1}}{\sqrt{2} \mu M_{2}} \tilde{W}^{-}-\frac{\mu_{1}}{\mu} \tilde{H}_{d}^{-}+\frac{\mu_{0}}{\mu} e_{1}, \\
e_{2}(\epsilon) & =e_{2} \\
e_{3}(\epsilon) & =e_{3}
\end{aligned}
$$

Notice that while the mixing between $e_{1}^{c}$ and $\tilde{H}_{u}^{+}$is tiny, the states $e_{1}$ and $\tilde{H}_{d}^{-}$have a large mixing angle, i.e. $\theta_{E}^{1}=\arctan \mu_{1} / \mu_{0} \approx 67^{\circ}$, for the required value of $y_{1}=\mu_{1} / \mu_{0}$ needed to fit the electron mass (cf. eq. (2.47)).

- Neutralino sector: for the eigenvalues we obtain

$$
\begin{aligned}
& \hat{M}_{n 1}(\epsilon)=M_{1}, \\
& \hat{M}_{n 2}(\epsilon)=M_{2}, \\
& \hat{M}_{n 3}(\epsilon)=-\mu, \\
& \hat{M}_{n 4}(\epsilon)=\mu \\
& \hat{m}_{\nu 1}(\epsilon)=-\frac{\mu_{1}^{2} v_{d}^{2}}{4 \mu^{2}}\left(\frac{g^{\prime 2}}{M_{1}}+\frac{g^{2}}{M_{2}}\right), \\
& \hat{m}_{\nu 2}(\epsilon)=0 \\
& \hat{m}_{\nu 3}(\epsilon)=0 .
\end{aligned}
$$

while, for the eigenstate associated with the massive neutrino (the remaining eigenstates are phenomenologically less important and can be easily inferred from the relevant mass matrix) we get

$$
\nu_{1}(\epsilon)=-\frac{g^{\prime} v_{d} \mu_{1}}{2 \mu M_{1}} \tilde{B}^{0}+\frac{g v_{d} \mu_{1}}{2 \mu M_{2}} \tilde{W}^{0}-\frac{\mu_{1}}{\mu} \tilde{H}_{d}^{0}+\frac{\mu_{0}}{\mu} \nu_{1} .
$$


The massive neutrino is hence maximally mixed with the neutral Higgsino. This is in full analogy with the electron-charged Higgsino mixing in eq. (A.17). In fact, the source of R-parity breaking $\mu_{1}$ is associated with an $\mathrm{SU}(2)$ invariant operator, so we expect the same large mixing for both the components of the $\mathrm{SU}(2)$ multiplets $L_{1}=\left(\nu_{1}, e_{1}\right)^{T}$ and $\tilde{H}_{d}=\left(\tilde{H}_{d}^{0}, \tilde{H}_{d}^{-}\right)^{T}$.

Open Access. This article is distributed under the terms of the Creative Commons Attribution License (CC-BY 4.0), which permits any use, distribution and reproduction in any medium, provided the original author(s) and source are credited.

\section{References}

[1] S. Dimopoulos and H. Georgi, Softly Broken Supersymmetry and SU(5), Nucl. Phys. B 193 (1981) 150 [INSPIRE].

[2] K.S. Babu, B. Bajc and Z. Tavartkiladze, Realistic Fermion Masses and Nucleon Decay Rates in SUSY SU(5) with Vector-Like Matter, Phys. Rev. D 86 (2012) 075005 [arXiv: 1207.6388] [INSPIRE].

[3] L.J. Hall, V.A. Kostelecky and S. Raby, New Flavor Violations in Supergravity Models, Nucl. Phys. B 267 (1986) 415 [InSPIRE].

[4] L.J. Hall, R. Rattazzi and U. Sarid, The top quark mass in supersymmetric $\mathrm{SO}(10)$ unification, Phys. Rev. D 50 (1994) 7048 [hep-ph/9306309] [INSPIRE].

[5] R. Hempfling, Yukawa coupling unification with supersymmetric threshold corrections, Phys. Rev. D 49 (1994) 6168 [INSPIRE].

[6] D.M. Pierce, J.A. Bagger, K.T. Matchev and R.-j. Zhang, Precision corrections in the minimal supersymmetric standard model, Nucl. Phys. B 491 (1997) 3 [hep-ph/9606211] [INSPIRE].

[7] F. Borzumati, G.R. Farrar, N. Polonsky and S.D. Thomas, Soft Yukawa couplings in supersymmetric theories, Nucl. Phys. B 555 (1999) 53 [hep-ph/9902443] [INSPIRE].

[8] S. Antusch and M. Spinrath, Quark and lepton masses at the GUT scale including SUSY threshold corrections, Phys. Rev. D 78 (2008) 075020 [arXiv:0804.0717] [INSPIRE].

[9] T. Enkhbat, SU(5) unification for Yukawas through SUSY threshold effects, arXiv:0909.5597 [INSPIRE].

[10] M. Iskrzynski, Effects of supersymmetric threshold corrections on the Yukawa matrix unification, Eur. Phys. J. C 75 (2015) 51 [arXiv:1408.2165] [INSPIRE].

[11] A. Anandakrishnan, B.C. Bryant and S. Raby, Threshold Corrections to the Bottom Quark Mass Revisited, JHEP 05 (2015) 088 [arXiv:1411.7035] [inSPIRE].

[12] M. Iskrzynski and K. Kowalska, Exact SU(5) Yukawa matrix unification in the General Flavour Violating MSSM, JHEP 04 (2015) 120 [arXiv:1412.8651] [INSPIRE].

[13] J.A. Casas, A. Lleyda and C. Muñoz, Strong constraints on the parameter space of the MSSM from charge and color breaking minima, Nucl. Phys. B 471 (1996) 3 [hep-ph/9507294] [INSPIRE].

[14] R. Barbier et al., R-parity violating supersymmetry, Phys. Rept. 420 (2005) 1 [hep-ph/0406039] [INSPIRE]. 
[15] A.Y. Smirnov and F. Vissani, Large R-parity violating couplings and grand unification, Nucl. Phys. B 460 (1996) 37 [hep-ph/9506416] [INSPIRE].

[16] M.A. Diaz, J. Ferrandis, J.C. Romao and J.W.F. Valle, Gauge and Yukawa unification with broken R-parity, Phys. Lett. B 453 (1999) 263 [hep-ph/9801391] [INSPIRE].

[17] H.K. Dreiner and H. Pois, Two loop supersymmetric renormalization group equations including R-parity violation and aspects of unification, hep-ph/9511444 [INSPIRE].

[18] E. Witten, Neutrino Masses in the Minimal O(10) Theory, Phys. Lett. B 91 (1980) 81 [INSPIRE].

[19] Z.G. Berezhiani, Horizontal Symmetry and Quark - Lepton Mass Spectrum: The $\mathrm{SU}(5) \times \mathrm{SU}(3)-h$ Model, Phys. Lett. B 150 (1985) 177 [INSPIRE].

[20] A. Davidson and K.C. Wali, $\mathrm{SU}(5)_{L} \otimes \mathrm{SU}(5)_{R}$ hybrid unification, Phys. Rev. Lett. 58 (1987) 2623 [INSPIRE].

[21] J. Hisano, H. Murayama and T. Yanagida, Double-triplet splitting in a supersymmetric SO(10) model without fine tuning, Phys. Rev. D 49 (1994) 4966 [INSPIRE].

[22] K.S. Babu and S.M. Barr, An $\mathrm{SO}(10)$ solution to the puzzle of quark and lepton masses, Phys. Rev. Lett. 75 (1995) 2088 [hep-ph/9503215] [INSPIRE].

[23] Z.G. Berezhiani, Predictive SUSY SO(10) model with very low tan Beta, Phys. Lett. B 355 (1995) 178 [hep-ph/9505384] [inSPIRE].

[24] K.S. Babu and S.M. Barr, Large neutrino mixing angles in unified theories, Phys. Lett. B 381 (1996) 202 [hep-ph/9511446] [INSPIRE].

[25] K.S. Babu and S.M. Barr, Realistic quark and lepton masses through $\mathrm{SO}(10)$ symmetry, Phys. Rev. D 56 (1997) 2614 [hep-ph/9512389] [INSPIRE].

[26] S.M. Barr and I. Dorsner, Explaining why the u and d quark masses are similar, Phys. Lett. B 566 (2003) 125 [hep-ph/0305090] [INSPIRE].

[27] M. Malinsky, Quark and lepton masses and mixing in $\mathrm{SO}(10)$ with a GUT-scale vector matter, Phys. Rev. D 77 (2008) 055016 [arXiv:0710.0581] [INSPIRE].

[28] Q. Shafi and Z. Tavartkiladze, An Improved supersymmetric SU(5), Phys. Lett. B 459 (1999) 563 [hep-ph/9904249] [INSPIRE].

[29] N. Oshimo, Realistic model for SU(5) grand unification, Phys. Rev. D 80 (2009) 075011 [arXiv:0907.3400] [INSPIRE].

[30] C.S. Aulakh and R.N. Mohapatra, Neutrino as the Supersymmetric Partner of the Majoron, Phys. Lett. B 119 (1982) 136 [INSPIRE].

[31] L.J. Hall and M. Suzuki, Explicit R-Parity Breaking in Supersymmetric Models, Nucl. Phys. B 231 (1984) 419 [INSPIRE].

[32] G.G. Ross and J.W.F. Valle, Supersymmetric Models Without R-Parity, Phys. Lett. B 151 (1985) 375 [INSPIRE].

[33] J.R. Ellis, G. Gelmini, C. Jarlskog, G.G. Ross and J.W.F. Valle, Phenomenology of Supersymmetry with Broken R-Parity, Phys. Lett. B 150 (1985) 142 [INSPIRE].

[34] A. Masiero and J.W.F. Valle, A Model for Spontaneous R Parity Breaking, Phys. Lett. B 251 (1990) 273 [INSPIRE]. 
[35] S. Davidson and M. Losada, Neutrino masses in the $R_{p}$ violating MSSM, JHEP 05 (2000) 021 [hep-ph/0005080] [INSPIRE].

[36] S. Davidson and M. Losada, Basis independent neutrino masses in the $R(p)$ violating MSSM, Phys. Rev. D 65 (2002) 075025 [hep-ph/0010325] [InSPIRE].

[37] M. Bisset, O.C.W. Kong, C. Macesanu and L.H. Orr, A simple phenomenological parametrization of supersymmetry without R-parity, Phys. Lett. B 430 (1998) 274 [hep-ph/9804282] [INSPIRE].

[38] P. Minkowski, $\mu \rightarrow e \gamma$ at a Rate of One Out of $10^{9}$ Muon Decays?, Phys. Lett. B 67 (1977) 421 [INSPIRE].

[39] T. Yanagida, Horizontal symmetry and masses of neutrinos, in proceedings of The Workshop on the Baryon Number of the Universe and Unified Theories, Tsukuba, Japan, 13-14 February 1979.

[40] M. Gell-Mann, P. Ramond and R. Slansky, Complex Spinors And Unified Theories, in Super-gravity, P. van Nieuwenhuizen and D.Z. Freedman eds., North Holland Publishing Co., (1979), [arXiv: 1306.4669] [INSPIRE].

[41] S.L. Glashow, The Future of Elementary Particle Physics, NATO Sci. Ser. B 61 (1979) 687 [INSPIRE].

[42] R.N. Mohapatra and G. Senjanović, Neutrino Mass and Spontaneous Parity Violation, Phys. Rev. Lett. 44 (1980) 912 [INSPIRE].

[43] R. Foot, H. Lew, X.G. He and G.C. Joshi, Seesaw Neutrino Masses Induced by a Triplet of Leptons, Z. Phys. C 44 (1989) 441 [inSPIRE].

[44] A.Y. Smirnov and F. Vissani, Upper bound on all products of R-parity violating couplings lambda-prime and lambda-prime-prime from proton decay, Phys. Lett. B 380 (1996) 317 [hep-ph/9601387] [INSPIRE].

[45] L. Di Luzio, M. Nardecchia and A. Romanino, Framework for baryonic R-parity violation in grand unified theories, Phys. Rev. D 88 (2013) 115008 [arXiv:1305.7034] [INSPIRE].

[46] S. Dimopoulos, S. Raby and F. Wilczek, Supersymmetry and the Scale of Unification, Phys. Rev. D 24 (1981) 1681 [INSPIRE].

[47] L.E. Ibáñez and G.G. Ross, Low-Energy Predictions in Supersymmetric Grand Unified Theories, Phys. Lett. B 105 (1981) 439 [InSPIRE].

[48] M.B. Einhorn and D.R.T. Jones, The Weak Mixing Angle and Unification Mass in Supersymmetric SU(5), Nucl. Phys. B 196 (1982) 475 [INSPIRE].

[49] W.J. Marciano and G. Senjanović, Predictions of Supersymmetric Grand Unified Theories, Phys. Rev. D 25 (1982) 3092 [InSPIRE].

[50] H. Murayama and A. Pierce, Not even decoupling can save minimal supersymmetric SU(5), Phys. Rev. D 65 (2002) 055009 [hep-ph/0108104] [InSPIRE].

[51] B. Bajc, P. Fileviez Perez and G. Senjanović, Proton decay in minimal supersymmetric SU(5), Phys. Rev. D 66 (2002) 075005 [hep-ph/0204311] [InSPIRE].

[52] B. Bajc, P. Fileviez Perez and G. Senjanović, Minimal supersymmetric SU(5) theory and proton decay: Where do we stand?, hep-ph/0210374 [INSPIRE].

[53] J. Hisano, D. Kobayashi, T. Kuwahara and N. Nagata, Decoupling Can Revive Minimal Supersymmetric SU(5), JHEP 07 (2013) 038 [arXiv: 1304.3651] [INSPIRE]. 
[54] B. Bajc, S. Lavignac and T. Mede, Challenging the minimal supersymmetric SU(5) model, AIP Conf. Proc. 1604 (2014) 297 [arXiv:1310.3093] [InSPIRE].

[55] J. Hisano, H. Murayama and T. Yanagida, Nucleon decay in the minimal supersymmetric SU(5) grand unification, Nucl. Phys. B 402 (1993) 46 [hep-ph/9207279] [INSPIRE].

[56] F. Vissani, $(B+L)$ conserving nucleon decays in supersymmetric models, Phys. Rev. D 52 (1995) 4245 [hep-ph/9503227] [INSPIRE].

[57] K.S. Babu and R.N. Mohapatra, B - L Violating Nucleon Decay and GUT Scale Baryogenesis in SO(10), Phys. Rev. D 86 (2012) 035018 [arXiv:1203.5544] [INSPIRE].

[58] M. Masip and A. Rasin, Minimal supersymmetric scenarios for spontaneous CP-violation, Phys. Rev. D 58 (1998) 035007 [hep-ph/9803271] [INSPIRE].

[59] M. Hirsch, M.A. Diaz, W. Porod, J.C. Romao and J.W.F. Valle, Neutrino masses and mixings from supersymmetry with bilinear $R$ parity violation: A theory for solar and atmospheric neutrino oscillations, Phys. Rev. D 62 (2000) 113008 [hep-ph/0004115] [INSPIRE].

[60] M.A. Diaz, M. Hirsch, W. Porod, J.C. Romao and J.W.F. Valle, Solar neutrino masses and mixing from bilinear $R$ parity broken supersymmetry: Analytical versus numerical results, Phys. Rev. D 68 (2003) 013009 [hep-ph/0302021] [INSPIRE].

[61] Y. Grossman and S. Rakshit, Neutrino masses in R-parity violating supersymmetric models, Phys. Rev. D 69 (2004) 093002 [hep-ph/0311310] [INSPIRE].

[62] M. Hirsch and J.W.F. Valle, Supersymmetric origin of neutrino mass, New J. Phys. 6 (2004) 76 [hep-ph/0405015] [INSPIRE].

[63] M. Bisset, O.C.W. Kong, C. Macesanu and L.H. Orr, Supersymmetry without R-parity: Leptonic phenomenology, Phys. Rev. D 62 (2000) 035001 [hep-ph/9811498] [INSPIRE].

[64] M. Nowakowski and A. Pilaftsis, $W$ and $Z$ boson interactions in supersymmetric models with explicit R-parity violation, Nucl. Phys. B 461 (1996) 19 [hep-ph/9508271] [InSPIRE].

[65] C. Frugiuele and T. Gregoire, Making the Sneutrino a Higgs with a $\mathrm{U}(1)_{R}$ Lepton Number, Phys. Rev. D 85 (2012) 015016 [arXiv:1107.4634] [INSPIRE].

[66] F. Riva, C. Biggio and A. Pomarol, Is the $125 \mathrm{GeV}$ Higgs the superpartner of a neutrino?, JHEP 02 (2013) 081 [arXiv:1211.4526] [INSPIRE].

[67] Particle Data Group collaboration, K.A. Olive et al., Review of Particle Physics, Chin. Phys. C 38 (2014) 090001.

[68] S. Davidson, S. Lacroix and P. Verdier, LHC sensitivity to lepton flavour violating Z boson decays, JHEP 09 (2012) 092 [arXiv: 1207.4894] [INSPIRE].

[69] W. Loinaz, N. Okamura, S. Rayyan, T. Takeuchi and L.C.R. Wijewardhana, The NuTeV anomaly, lepton universality and nonuniversal neutrino gauge couplings, Phys. Rev. D 70 (2004) 113004 [hep-ph/0403306] [INSPIRE].

[70] W.-Y. Keung and G. Senjanović, Majorana Neutrinos and the Production of the Right-handed Charged Gauge Boson, Phys. Rev. Lett. 50 (1983) 1427 [InSPIRE].

[71] B. Bajc and G. Senjanović, Seesaw at LHC, JHEP 08 (2007) 014 [hep-ph/0612029] [INSPIRE].

[72] B. Bajc, M. Nemevšek and G. Senjanović, Probing seesaw at LHC, Phys. Rev. D 76 (2007) 055011 [hep-ph/0703080] [INSPIRE]. 
[73] A. Arhrib et al., Collider Signatures for Heavy Lepton Triplet in Type I+III Seesaw, Phys. Rev. D 82 (2010) 053004 [arXiv: 0904.2390] [InSPIRE].

[74] L. Di Luzio and L. Mihaila, Unification scale vs. electroweak-triplet mass in the SU(5) $+24_{F}$ model at three loops, Phys. Rev. D 87 (2013) 115025 [arXiv: 1305.2850] [InSPIRE].

[75] J.E. Kim, P. Ko and D.-G. Lee, More on R-parity and lepton family number violating couplings from muon(ium) conversion and tau and pi0 decays, Phys. Rev. D 56 (1997) 100 [hep-ph/9701381] [INSPIRE].

[76] A. de Gouvêa, S. Lola and K. Tobe, Lepton flavor violation in supersymmetric models with trilinear R-parity violation, Phys. Rev. D 63 (2001) 035004 [hep-ph/0008085] [INSPIRE].

[77] R. Kitano, M. Koike and Y. Okada, Detailed calculation of lepton flavor violating muon electron conversion rate for various nuclei, Phys. Rev. D 66 (2002) 096002 [hep-ph/0203110] [INSPIRE].

[78] SINDRUM II collaboration, C. Dohmen et al., Test of lepton flavor conservation in $\mu \rightarrow e$ conversion on titanium, Phys. Lett. B 317 (1993) 631 [INSPIRE].

[79] B. Bajc, T. Enkhbat, D.K. Ghosh, G. Senjanović and Y. Zhang, MSSM in view of PAMELA and Fermi-LAT, JHEP 05 (2010) 048 [arXiv:1002.3631] [INSPIRE].

[80] F. Takayama and M. Yamaguchi, Gravitino dark matter without R-parity, Phys. Lett. B 485 (2000) 388 [hep-ph/0005214] [INSPIRE].

[81] U. Oberlack, Viewpoint: Extragalactic diffuse gamma-ray emission at high energies, Physics 3 (2010) 21, http://physics.aps.org/articles/v3/21.

[82] Fermi-LAT collaboration, A.A. Abdo et al., The Spectrum of the Isotropic Diffuse Gamma-Ray Emission Derived From First-Year Fermi Large Area Telescope Data, Phys. Rev. Lett. 104 (2010) 101101 [arXiv:1002.3603] [INSPIRE].

[83] R. Essig, E. Kuflik, S.D. McDermott, T. Volansky and K.M. Zurek, Constraining Light Dark Matter with Diffuse X-Ray and Gamma-Ray Observations, JHEP 11 (2013) 193 [arXiv: 1309.4091] [INSPIRE].

[84] J.W. den Herder et al., The search for decaying Dark Matter, arXiv:0906.1788 [INSPIRE].

[85] J. Pradler and F.D. Steffen, Thermal gravitino production and collider tests of leptogenesis, Phys. Rev. D 75 (2007) 023509 [hep-ph/0608344] [INSPIRE].

[86] V.S. Rychkov and A. Strumia, Thermal production of gravitinos, Phys. Rev. D 75 (2007) 075011 [hep-ph/0701104] [INSPIRE].

[87] M. Olechowski, S. Pokorski, K. Turzynski and J.D. Wells, Reheating Temperature and Gauge Mediation Models of Supersymmetry Breaking, JHEP 12 (2009) 026 [arXiv: 0908.2502] [INSPIRE].

[88] G. Arcadi, L. Di Luzio and M. Nardecchia, Gravitino Dark Matter in Tree Level Gauge Mediation with and without R-parity, JHEP 12 (2011) 040 [arXiv:1110.2759] [INSPIRE].

[89] T.E. Clark, T.-K. Kuo and N. Nakagawa, A SO(10) supersymmetric grand unified theory, Phys. Lett. B 115 (1982) 26 [InSPIRE].

[90] C.S. Aulakh and R.N. Mohapatra, Implications of Supersymmetric $\mathrm{SO}(10)$ Grand Unification, Phys. Rev. D 28 (1983) 217 [INSPIRE].

[91] C.S. Aulakh, B. Bajc, A. Melfo, G. Senjanović and F. Vissani, The minimal supersymmetric grand unified theory, Phys. Lett. B 588 (2004) 196 [hep-ph/0306242] [INSPIRE]. 
[92] R.N. Mohapatra, New Contributions to Neutrinoless Double beta Decay in Supersymmetric Theories, Phys. Rev. D 34 (1986) 3457 [InSPIRE].

[93] A. Font, L.E. Ibáñez and F. Quevedo, Does Proton Stability Imply the Existence of an Extra Z0?, Phys. Lett. B 228 (1989) 79 [inSPIRE].

[94] S.P. Martin, Some simple criteria for gauged R-parity, Phys. Rev. D 46 (1992) 2769 [hep-ph/9207218] [INSPIRE].

[95] C.S. Aulakh, K. Benakli and G. Senjanović, Reconciling supersymmetry and left-right symmetry, Phys. Rev. Lett. 79 (1997) 2188 [hep-ph/9703434] [INSPIRE].

[96] C.S. Aulakh, A. Melfo and G. Senjanović, Minimal supersymmetric left-right model, Phys. Rev. D 57 (1998) 4174 [hep-ph/9707256] [INSPIRE].

[97] C.S. Aulakh, A. Melfo, A. Rasin and G. Senjanović, Seesaw and supersymmetry or exact R-parity, Phys. Lett. B 459 (1999) 557 [hep-ph/9902409] [INSPIRE].

[98] S.R. Coleman and E.J. Weinberg, Radiative Corrections as the Origin of Spontaneous Symmetry Breaking, Phys. Rev. D 7 (1973) 1888 [inSPIRE].

[99] B. Bajc and A. Melfo, Metastable gauged O'Raifeartaigh, JHEP 04 (2008) 062 [arXiv: 0801.4349] [INSPIRE].

[100] B. Bajc, S. Lavignac and T. Mede, Supersymmetry Breaking Induced by Radiative Corrections, JHEP 07 (2012) 185 [arXiv: 1202.2845] [INSPIRE].

[101] G.F. Giudice and R. Rattazzi, Extracting supersymmetry breaking effects from wave function renormalization, Nucl. Phys. B 511 (1998) 25 [hep-ph/9706540] [INSPIRE].

[102] S.P. Martin, Non-universal gaugino masses from non-singlet F-terms in non-minimal unified models, Phys. Rev. D 79 (2009) 095019 [arXiv:0903.3568] [InSPIRE]. 